\title{
Aeroelastic Indicial Response Reduced-Order Modeling for Flexible Flight Vehicles
}

\author{
Brett R. Hiller*, Neal T. Frink ${ }^{\dagger}$, Walter A. Silva ${ }^{\ddagger}$ \\ NASA Langley Research Center, Hampton, VA, 23681 \\ Dimitri N. Mavris $\$$ \\ Aerospace Systems Design Laboratory, Georgia Institute of Technology, Atlanta, GA, 30332
}

\begin{abstract}
A reduced-order modeling method capable of providing computationally efficient predictions of the nonlinear, unsteady aerodynamics encountered by flexible flight vehicles undergoing forced oscillations is presented. Models are developed using indicial response theory, which characterizes a vehicle's dynamics through identification of time-accurate aerodynamic responses due to step changes in the vehicle-state parameters, e.g., angle-of-attack, pitch rate. A coupled computational fluid dynamics aeroelastic analysis is proposed for identifying step (indicial) responses of flexible vehicles. In this approach, aeroelastic indicial responses are simulated via prescribed rigid body motions, while fluid-structure interactions are captured at the subiterative level through coupling to a linear modal structural solver. A nonlinear extension of indicial response theory is applied through time-dependent linear interpolation of a database of locally linear aeroelastic step responses. Reduced-order models are then created using the mathematical principle of convolution applied to the interpolated aeroelastic indicial responses to predict the time-dependent aerodynamic response of a flexible vehicle to any arbitrary prescribed flight maneuver. The NASA FUN3D computational fluid dynamics solver is utilized for simulating full-order trajectories and indicial response functions. Aerodynamic predictions were generated for the $\mathrm{X}-56 \mathrm{~A}$ aircraft undergoing a series of harmonic forced oscillations. The reduced-order modeling solutions are shown to provide a practical option for evaluating the unsteady aerodynamics of flexible vehicles using high-fidelity simulations.
\end{abstract}

\begin{tabular}{|c|c|c|c|c|}
\hline \multicolumn{5}{|c|}{ Nomenclature } \\
\hline$c_{r e f}$ & $=$ & wing chord, in & $p$ & $=$ roll rate, $\mathrm{rad} / \mathrm{s}$ \\
\hline$b_{\text {ref }}$ & $=$ & wingspan, in & $q$ & pitch rate, $\mathrm{rad} / \mathrm{s}$ \\
\hline$S$ & $=$ & planform area, $\mathrm{in}^{2}$ & $r$ & $=$ yaw rate, $\mathrm{rad} / \mathrm{s}$ \\
\hline $\mathbf{x}_{M}$ & $=$ & moment center, in & $t_{f}$ & $=$ final maneuver time, $\mathrm{s}$ \\
\hline $\mathbf{L}_{M}$ & $=$ & moment lengths, in & $n_{s u b}$ & $=$ number of subiterations \\
\hline $\mathbf{x}_{C G}$ & $=$ & center of gravity, in & $n_{f s i}$ & number of aeroelastic coupling iterations \\
\hline$W$ & $=$ & weight, lb & $C_{L}$ & $=$ lift coefficient \\
\hline$\alpha$ & $=$ & angle-of-attack, deg & $C_{m}$ & $=$ pitching moment coefficient \\
\hline$\beta$ & $=$ & sideslip angle, deg & $C_{Y}$ & $=$ side force coefficient \\
\hline$M$ & $=$ & Mach number & $C_{l}$ & $=$ rolling moment coefficient \\
\hline$\phi$ & $=$ & roll angle, deg & $C_{n}$ & $=$ yawing moment coefficient \\
\hline$\theta$ & $=$ & pitch angle, deg & $f$ & $=$ frequency, $\mathrm{Hz}$ \\
\hline$\psi$ & $=$ & yaw angle, deg & $k$ & $=$ reduced frequency, $2 \pi f L_{r e f} / U_{\infty}$ \\
\hline$V$ & $=$ & velocity, in/s & $t$ & $=$ time, $\mathrm{s}$ \\
\hline$L_{r e f}$ & $=$ & reference length, in & $s$ & $=$ normalized time, $V t / c_{r e f}$ \\
\hline$N$ & $=$ & sampling resolution & & \\
\hline
\end{tabular}

\footnotetext{
* Research Student Trainee (PhD Candidate, Georgia Tech), Configuration Aerodynamics Branch, AIAA Student Member

${ }^{\dagger}$ Retired. Distinguished Research Associate, Configuration Aerodynamics Branch, AIAA Associate Fellow

$\doteqdot$ Senior Research Scientist, Aeroelasticity Branch, AIAA Associate Fellow

${ }^{\S}$ S.P. Langley NIA Distinguished Regents Professor, Daniel Guggenheim School of Aerospace Engineering, AIAA Fellow
} 


\section{Introduction}

Accurate aerodynamic predictions remain a cornerstone of the aircraft design process due to their significance in determining the performance and stability and control (S\&C) characteristics of aircraft. Traditional flight dynamics modeling has historically relied on the use of quasisteady stability derivatives, often calculated using simplified linear aerodynamic methods. These models are inherently incapable of predicting the nonlinear, unsteady aerodynamics encountered by modern flexible aircraft. Inaccurate predictions of such phenomena can lead to suboptimal vehicle performance and/or inaccurate control law design, potentially leading to loss of control of the vehicle.

Recent advances in digital computing have motivated interest toward Virtual Flight Simulation (VFS), for which multidisciplinary numerical simulations are used to evaluate an aircraft's performance and S\&C characteristics at full-scale flight Reynolds and Mach numbers [1, 2]. Despite their demonstrated feasibility, these simulations often require thousands of computational hours, limiting their adoption and widespread application for aircraft design and analysis.

Reduced-order modeling is viewed as a key enabler for the viable application of VFS methods. Reduced-order models (ROMs) are mathematical models aimed at accurately predicting the fundamental dynamics of a system at a computational cost much less than what is required in solving the original governing equations. These methods approximate the full-order numerical simulations of a system by extracting and reconstructing pertinent dynamic data solutions, without making any limiting physical modeling assumptions.

Significant progress in reduced-order modeling has been made over the past two decades with the development of a variety of reduced-order models for efficient unsteady aerodynamic predictions [3-7]. However, most unsteady aerodynamic ROMs have been primarily used for either predicting the aerodynamic response of rigid maneuvering vehicles or identifying aeroelastic instabilities, such as a flutter. Multidisciplinary ROMs for virtual flight simulations remain a desirable, yet relatively unexplored area of research.

This paper demonstrates a multidisciplinary ROM capable of providing quantitatively accurate, yet computationally efficient predictions of the nonlinear, unsteady aerodynamics encountered by maneuvering, flexible flight vehicles. Reduced-order models are constructed based on aerodynamic indicial response theory, which characterizes a system's dynamics through identification of the system (indicial) response due to numerically simulated unit step changes in a system's inputs. A coupled computational fluid dynamics aeroelastic analysis is proposed for identifying step (indicial) responses of flexible vehicles.

In this approach, aeroelastic indicial responses are simulated via prescribed rigid body motions, which isolate the effects of angle-of-attack and sideslip angle from angular rates, while fluid-structure interactions are captured at the subiterative level through coupling to a linear modal structural solver. A nonlinear extension of indicial response theory is evaluated through application of time-dependent linear interpolation to a database of locally linear step responses, simulated at various angles of attack defining the flight maneuver space. Reduced-order models are then created by applying the mathematical principle of convolution to interpolated aeroelastic indicial responses to predict the time-dependent aerodynamic response of a flexible vehicle to any arbitrary prescribed flight maneuver.

\section{Geometry}

The X-56A configuration is a lightweight, flexible aircraft developed by the U.S. Air Force Research Laboratory (AFRL) to serve as a Multi-Utility Technology Testbed (MUTT). An X-56A illustration is given in Fig. 1 The primary goals of the X-56A efforts were to investigate active flutter suppression technology and active shape control. To date, several topics of research have been published and include: the stability of closed-loop flutter wing models [8, 9]; stabilizing and controlling wing shape [10]; fly-by-feel sensing and control [11]; airframe structural optimization [12, 13]; and most recently, CFD-based dynamic aeroelastic analysis for flutter prediction [14]. All of these research topics rely on a structural model of the aircraft that has been verified/validated with ground vibration test results [15].

The aircraft consists of a rigid center body and flexible detachable wings, allowing for different structural properties and sensor packages to be tested. Additional details for the design and development of the X-56A can be found in the work by Beranek et al. [16] and Nicolai et al. [17]. The size, geometric quantities, and weight of the X-56A are summarized in Table 1. The present work leverages a set of structural mode shapes extracted from a finite-element structural model provided by Reasor et al. [14]. A single Euler grid is generated for comparing the aeroelastic ROM predictions to the full-order CFD simulation results. The engines, control surfaces, and landing gear are not included in the CFD grid for this work, as the primary focus is on modeling and simulating a flexible vehicle undergoing prescribed flight test maneuvers without propulsion or flight mechanics coupling. It is important to note, however, that the engine mounts, control surfaces, and landing gear are present in the structural mode shapes. 


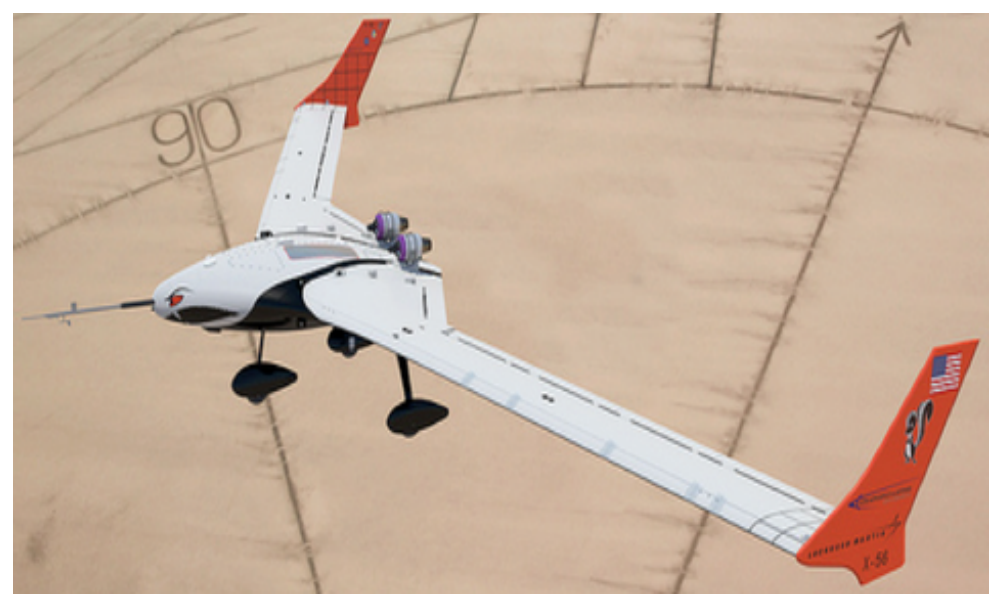

Figure 1. X-56A multi-utility technology testbed (Figure Courtesy: Lockheed Martin).

Table 1. Dimensions and mass properties of the X-56A configuration as simulated [16].

\begin{tabular}{lcrl} 
Description & Symbol & Value & Units \\
\hline Chord Length & $c_{\text {ref }}$ & 24.0 & in \\
Wingspan & $b_{\text {ref }}$ & 336.0 & in \\
Planform Area & $S$ & $11,088.0$ & in $^{2}$ \\
Moment Center & $\mathbf{x}_{M}$ & $\{165.0,0.0,101.3\}$ & in \\
Moment Lengths & $\mathbf{L}_{M}$ & $\{336.0,24.0,336.0\}$ & in \\
Center of Gravity & $\mathbf{x}_{C G}$ & $\{163.3,0.2311,101.3\}$ & in \\
Weight & $W$ & 495.6 & lb \\
\hline
\end{tabular}

\section{Computational Tools}

FUN3D v13.3 is a parallelized, unstructured, node-based, finite-volume discretization, RANS flow solver [18]. Inviscid flux quantities are computed using a variety of upwind flux functions, where the Roe flux construction scheme was utilized in the present work. Turbulence models currently available include the Spalart-Almaras (SA) model, Menter k-omega Shear Stress Transport (SST) model, Wilcox k-omega model, and detached eddy simulations, among others. Time-accurate simulations may be advanced in time using up to 3rd-order time step schemes, where pseudotime advancement is dictated by a linear CFL-subiteration schedule within each iteration. An optimized second-order backward differencing (BDF2opt) scheme was utilized to perform time-accurate static and dynamic Euler simulations of vehicle maneuver and indicial response trajectories.

A general prescribed rigid-body motion capability is available that relies on a 4 x 4 transformation matrix, specified at each point in discretized time, which maps the moving body coordinates to the fixed initial reference frame [19]. Prescribed trajectories are defined in terms of velocity components (angle-of-attack - $\alpha$, sideslip angle - $\beta$, and Mach number $-M$ ) and Euler angles (roll $-\phi$, pitch $-\theta$, and yaw $-\psi$ ). The general procedure for simulating vehicle and indicial response trajectories in FUN3D is implemented as described in the authors' previous work [20].

In addition to its baseline solver capabilities, FUN3D has been extended to handle general mesh movement involving rigid and deforming meshes [19]. FUN3D's aeroelastic module provides the capability for performing static and dynamic aeroelastic analyses via time integration of the linear structural dynamics equations. The static aeroelastic analysis is useful for problems in which the coupling between the aerodynamics and structures disciplines is relatively weak and may be done infrequently, e.g., cruise conditions. Whereas, the dynamic analysis is useful for problems involving a strong, unsteady coupling between the two disciplines.

As opposed to externally coupling FUN3D's CFD solver with a nonlinear Finite Element Method (FEM) model, FUN3D uses a self-contained, mode-based linear structural model. The modal aeroelastic analysis formulation is capable of predicting the fluid-structure interactions (FSI) for models subject to small or moderate surface deflections, and its use has become routine for flutter predictions. Details of the modal aeroelastic analysis and time integration scheme may be found in Ref. [19]. 
Prior work demonstrated the implementation of FUN3D's rigid-body motion capability in simulating a rigid, unmanned combat air vehicle performing a series of forced oscillations, a half Lazy- 8 turn maneuver, and an Immelmann turn maneuver [20]. The present work serves as the first demonstration of FUN3D's capability for performing dynamic aeroelastic simulations of flexible vehicles undergoing prescribed flight trajectories. Figure 2 outlines the dynamic aeroelastic simulation process for generating indicial response and maneuver solutions. For a given trajectory, a wellconverged, static time-accurate aeroelastic solution is first generated at the initial flight condition $\left(\alpha_{0}, \beta_{0}, M_{0}\right)$. The vehicle velocity components $(\alpha(t), \beta(t), M(t))$ and Euler angles $(\phi(t), \theta(t), \psi(t))$ are mapped to translations $(X(t), Y(t), Z(t))$ relative to the inertial reference frame at each point in the trajectory. The calculated vehicle translations and rotations are then used to create a FUN3D motion history file, defined by a series of $4 \times 4$ transformation matrices for each trajectory time step. A summary of this procedure is given in Frink et al. [20]. A time-accurate dynamic aeroelastic simulation is then initialized from the static aeroelastic solution and advanced in time using a specified time step size until the maneuver is complete at a final time $\left(t_{f}\right)$. For each step in time, a set number of subiterations $\left(n_{\text {sub }}\right)$ are used to converge the fluid dynamic state due to the change in vehicle-state at each time instance along the trajectory. These converged aerodynamic loads serve as inputs to FUN3D's mode-based linear structural model, which then solves for the resulting vehicle deformation. Finally, the CFD mesh is deformed by solving the linear elasticity equations for mesh deformation. The coupling between the flow solver and structural solver may be repeated up to a prescribed number of iteratations $\left(n_{f s i}\right)$.

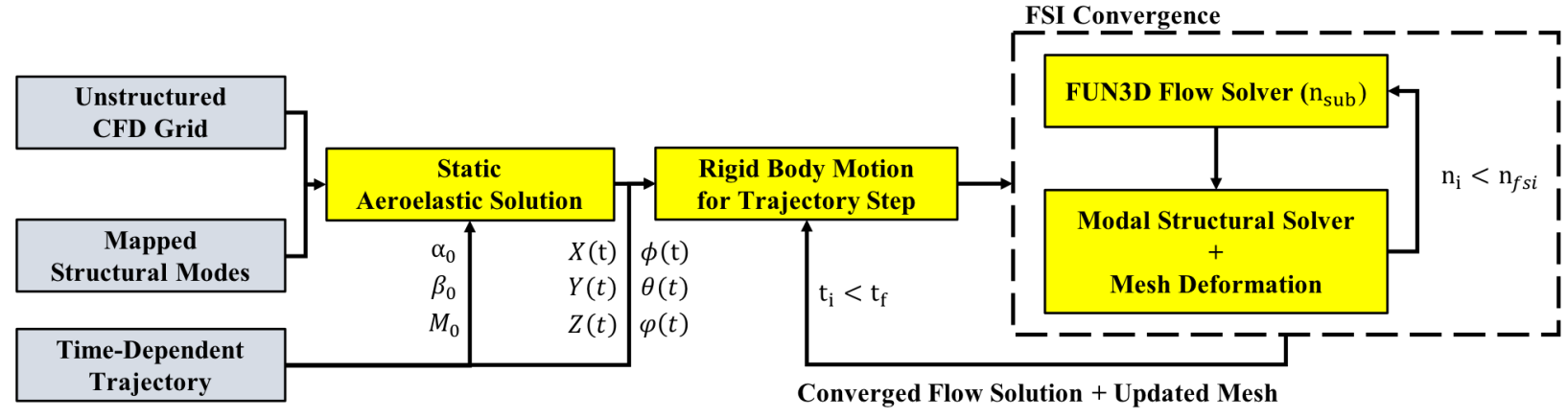

Figure 2. Dynamic aeroelastic simulation process.

\section{Grid Generation}

The grid used for the present work was generated with Pointwise V18.0 using IGS formatted computer-aided-design (CAD) geometry, provided by Christopher Acuff of the NASA Armstrong Flight Research Center, exported from SolidWorks 2014. The X-56A model consists solely of the center body and flexible wings with the landing gear, control surfaces, and engines removed as means of reducing computational costs and mesh deformation complexity.

A single mixed-element, coarse grid was generated for the dynamic coupled Euler-aeroelastic analyses and consists of 579,506 total nodes, of which 74,914 were surface nodes. The resulting surface resolution is shown in a top semispan view in Fig. 3(a) Both the leading and trailing edges of the configuration were resolved using Pointwise's T-Rex cells, which consisted of layers of anisotropic quadrilateral cells emanating from the boundary curves. Figure $3(\mathrm{~b})$ illustrates the resulting layers in a closeup view of the center body and leading edge on the left wing. The initial spacing and growth rate for each of the layers was specified as $0.1 \mathrm{in}$. and $20 \%$, respectively, where the initial spacing is approximately $20 \%$ of the trailing edge thickness.

The computational domain was generated using a 'sphere primitive' centered at the model moment center with a radius of 100 chord-lengths (2,400 in). Figure 4 illustrates the growth of the grid domains at the $y=0$ plane, where Fig. 4(a) focuses on the domain growth near the center body profile, and Fig. 4(b) shows the resolution extending to the spherical farfield domain. In its development, the grid was first generated as a hemisphere volume with an $\mathrm{x}-\mathrm{z}$ symmetry plane, and subsequently, copied and mirrored about the symmetry plane to yield a symmetric volume grid. While not completely essential for a CFD-to-ROM comparison, this is a desirable practice when simulating complex six-degree-of-freedom (6DOF) trajectories. For the CFD boundary conditions, the vehicle surface was set as the zero normal velocity tangency condition (3000), and the farfield was set as the external freestream condition (5050). 


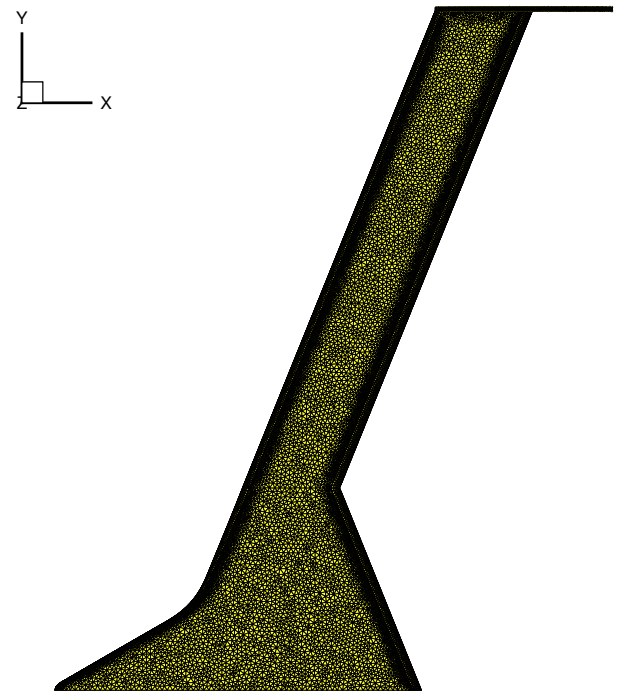

(a) Top view of semispan wing

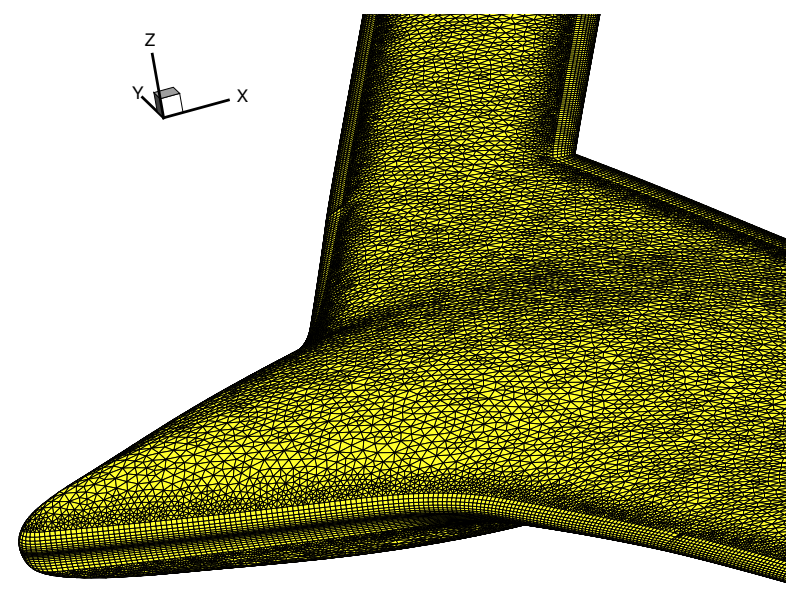

(b) Closeup of center body and leading edge

Figure 3. X-56A grid surface resolution.

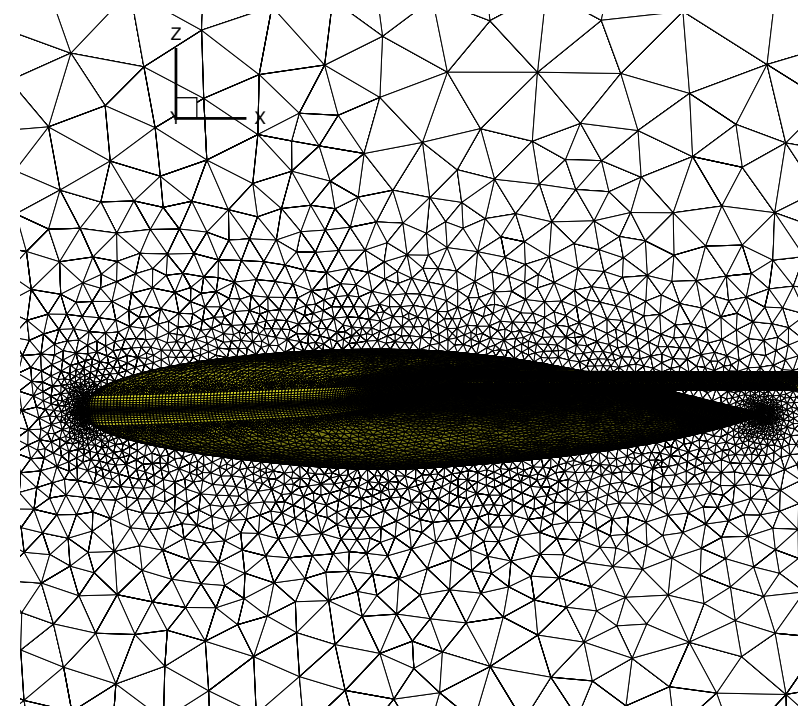

(a) Near body

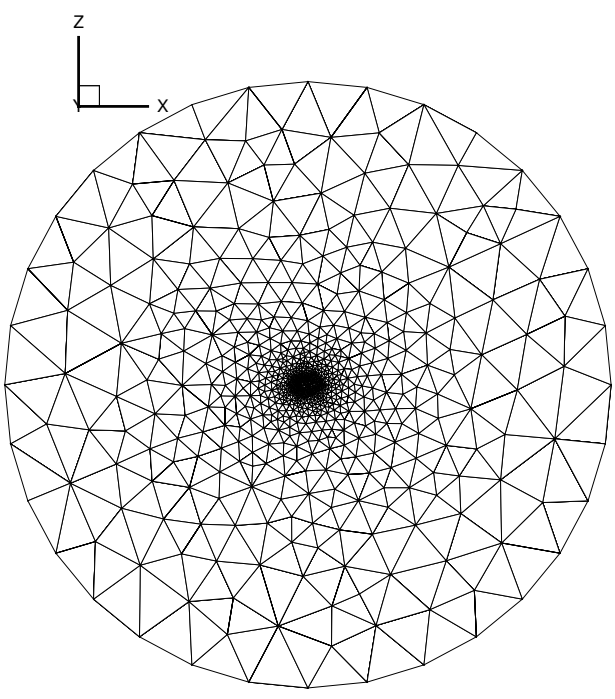

(b) Spherical farfield

Figure 4. X-56A grid domain growth. 


\section{Structural Modeling}

The structural model used in this work was originally developed at Lockheed Martin Skunkworks and further validated and calibrated to match ground vibration test results from NASA Armstrong [15, 21]. It is a finite element model (FEM) constructed in NASTRAN using 8,249 nodes. To take advantage of the modal structural dynamics solver within FUN3D, a linear modal solution is used in the present work instead of relying on an externally coupled simulation to the high-fidelity FEM model. The full modal solution contains 6 rigid-body modes and 34 elastic modes with a range from 3-60 Hz where numerous modes contain control surface deflections. Pak \& Truong [15] found the first four elastic modes to be the most critical to predicting the three flutter modes of the X-56A.

The present work incorporates four primary modes for high-fidelity maneuvering vehicle aeroelastic simulations as a means of reducing computational costs. It is important to note that the engine mounts, control surfaces, and landing gear are present in the structural mode shapes, but are not modeled in the CFD grids to further simplify simulations. The four elastic modes correspond to symmetric wing first bending (SW1B), antisymmetric wing first bending (AW1B), symmetric wing first torsion (SW1T), and antisymmetric wing first torsion (AW1T), as shown in Fig. 5
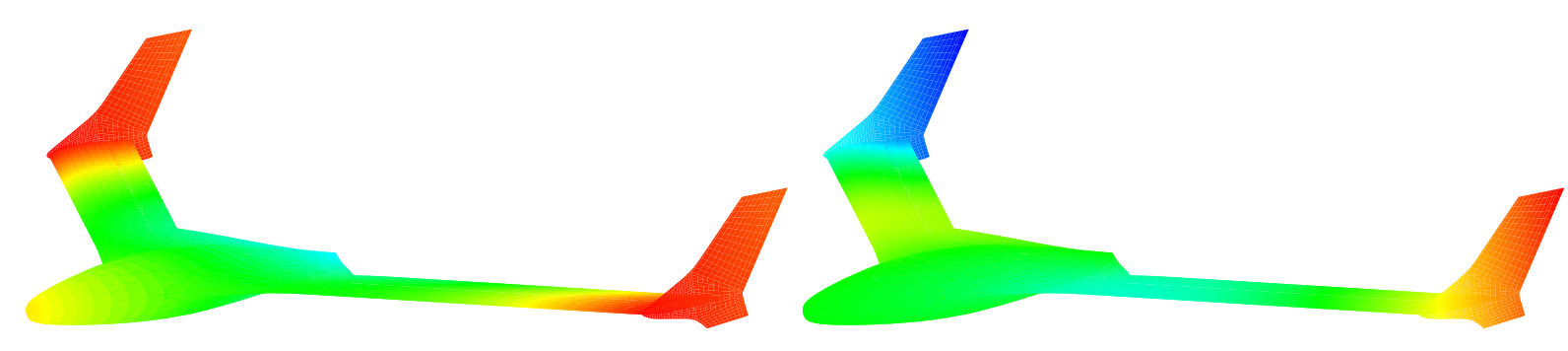

(a) Mode 1 - Symmetric Wing First Bending (SW1B) - $3.06 \mathrm{~Hz}$

(b) Mode 2 - Antisymmetric Wing First Bending (AW1B) - $4.85 \mathrm{~Hz}$
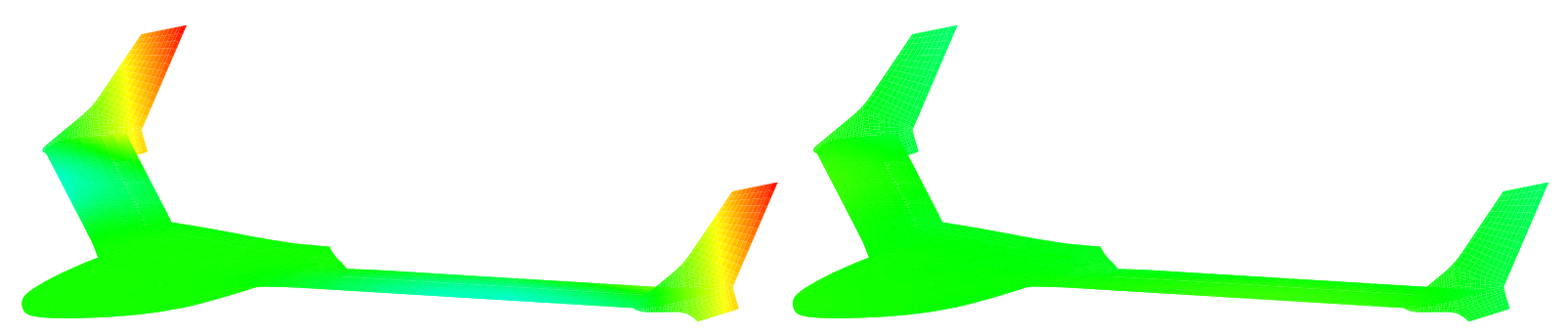

(c) Mode 3 - Symmetric Wing First Torsion (SW1T) - $11.09 \mathrm{~Hz}$

(d) Mode 4 - Antisymmetric Wing First Torsion (AW1T) - $11.95 \mathrm{~Hz}$

Figure 5. Four primary flexible modes of the X-56A. 


\section{Reduced-Order Modeling}

\section{A. Aerodynamic Indicial Response Theory}

Indicial response theory may be used to model any dynamic system that is classified as linear and time-invariant (TI). A time-invariant system is one whose fundamental properties do not change with time. This classification describes the system itself and makes no inference to the inputs or outputs of the system. An indicial response is defined as a system's output response, $y_{u}$, due to a unit step change in a system's input, $u$, as shown in Fig. 6. Indicial response theory assumes that a system's response can be linearized with respect to a system's forcing function. Under this assumption, the mathematical principle of convolution is used to predict the system response to any arbitrary time-varying input via the superposition of scaled and time-shifted indicial responses.

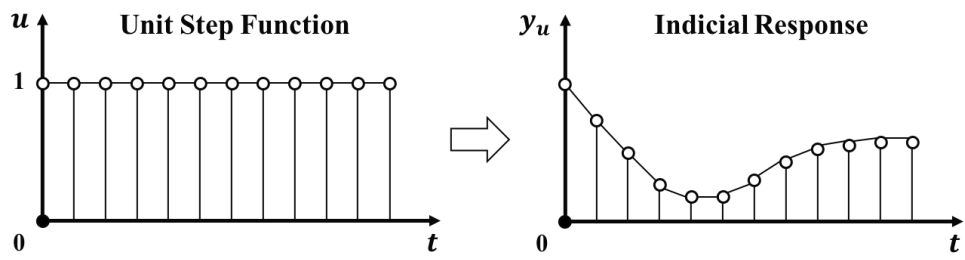

Figure 6. System's indicial (step) response due to a unit step in a system's input.

Equation 1 expresses the general form of an indicial response ROM used to predict a time-varying system output, $y(t)$, due to time-history contributions from some arbitrary time-varying system input, $u(t)$, as calculated by the convolution of the indicial response, $y_{u}(t)$, with the time-derivative of the given input, $\dot{u}(t)$. The first term, $y_{0}$, represents the initial output value, whereas $u(0)$ is the value of the system input at $t=0$. Using the differential theory of the convolution integral, the expression given on the right hand side recasts the convolution integral in terms of the indicial response and the system input.

$$
y(t)=y_{0}+u(0) y_{u}(t)+\int_{0}^{t} y_{u}(t-\tau) \frac{d u(\tau)}{d t} d \tau=y_{0}+\frac{d}{d t}\left[\int_{0}^{t} y_{u}(t-\tau) u(\tau) d \tau\right]
$$

Figure 7 provides an illustrative example of this convolution process. The input function linearly ramps over time, where its first-order time-derivative is represented by a step function. At each time, the system response to the linear ramping of the input $(u)$ is calculated via convolution. Simply put, convolution is the summation of indicial response functions, first initialized at each point in time and then scaled by the local value of the input derivative. This process effectively models the influence of previous system input states on the system output at any later time, i.e., an unsteady response to any arbitrary time-varying input.

Initial efforts in applying indicial response theory for unsteady aerodynamics modeling for maneuvering flight vehicles are detailed by Tobak et al. [22, 23] and Reisenthal et al. [24, 25]. Using the same assumed dependent vehiclestate parameters as the quasisteady stability derivative method, the unsteady aerodynamic response to longitudinal and/or lateral motions can be modeled within linear flight regimes using indicial response theory. Equation 2 expresses the unsteady lift and pitching moment, $C_{j}=\left[C_{L}, C_{m}\right]$, as a function of the convolution integrals with respect to any arbitrary time-varying maneuver defined by angle-of-attack, $\alpha$, and pitch rate, $q$. In Eq. 2, $C_{j_{0}}$ denotes the zero-angle-of-attack lift or pitching moment coefficients. The terms $C_{j_{\alpha}}$ and $C_{j_{q}}$ represent the transient indicial responses of the system with respect to unit step changes in angle-of-attack and pitch rate, respectively. Equation 3 expresses the unsteady side force, rolling moment, and yawing moment, $C_{k}=\left[C_{Y}, C_{l}, C_{n}\right.$, ], as a function of the convolution integrals with respect to any arbitrary time-varying maneuver defined by sideslip angle, $\beta$, roll rate, $p$, and yaw rate, $r$.

$$
\begin{gathered}
C_{j}(t)=C_{j_{0}}+\frac{d}{d t}\left[\int_{0}^{t} C_{j_{\alpha}}(t-\tau) \alpha(\tau) d \tau\right]+\frac{d}{d t}\left[\int_{0}^{t} C_{j_{q}}(t-\tau) q(\tau) d \tau\right] \\
C_{k}(t)=\frac{d}{d t}\left[\int_{0}^{t} C_{k_{\beta}}(t-\tau) \beta(\tau) d \tau\right]+\frac{d}{d t}\left[\int_{0}^{t} C_{k_{p}}(t-\tau) p(\tau) d \tau\right]+\frac{d}{d t}\left[\int_{0}^{t} C_{k_{r}}(t-\tau) r(\tau) d \tau\right]
\end{gathered}
$$




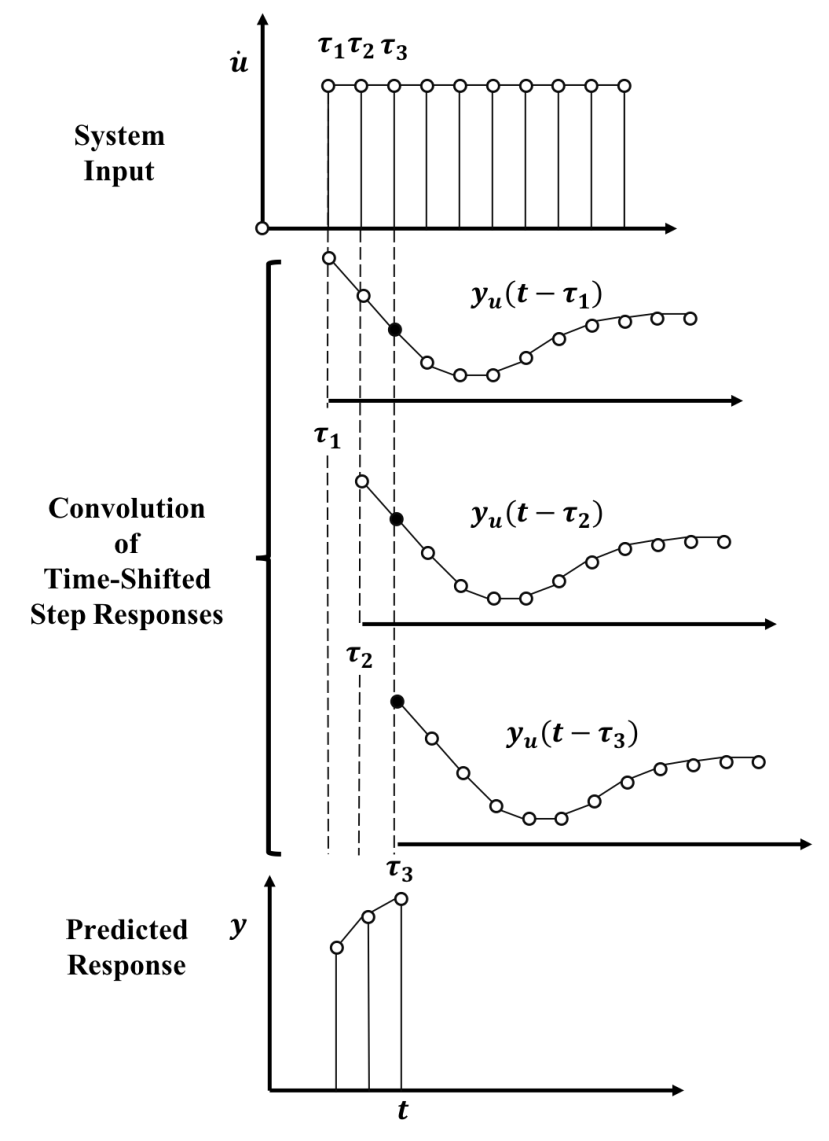

Figure 7. Convolution of indicial responses for ROM predictions.

Indicial responses are difficult to directly measure experimentally due to the inability to create an instantaneous step motion. Historically, indicial responses were identified either analytically, for 2D configurations, or experimentally, in conjunction with system identification methods. In contrast, numerical simulations are capable of simulating such impulsive changes as the governign equations are solved in discrete-time (DT). First observed by Silva [26], the discretized Navier-Stokes equations can be characterized as a discrete-time, nonlinear, time-invariant system of equations. These characteristics satisfy the requirements for indicial response theory system modeling. As such, discrete-time unit step and impulse functions have been identified and successively used in reduced-order models to characterize the dynamics for a variety of computational aerodynamic systems, particularly in the field of aeroelasticity [26-28]. More recently, Ghoreyshi et al. [29] leveraged the grid motion capabilities of modern CFD solvers to enable direct identification of indicial responses with respect to the trajectory-based parameters of Eqs. 2 and 3 for unsteady aerodynamic predictions of maneuvering aircraft. Using the procedure previously described for simulating rigid-body motions, indicial response trajectories may be defined that prescribe unit steps in angle-of-attack, sideslip angle, pitch rate, roll rate, or yaw rate independently while maintaining a constant value for the other parameters.

Figures 8 and 9 illustrate an example of CFD-calculated indicial responses with respect to step changes in angleof-attack and pitch rate. In this approach, a step change in angle-of-attack is achieved by specifying a grid motion, beginning at $t=0$, consisting of a translation aft and downward. During this motion, changes in the aerodynamic loads are isolated to contributions from angle-of-attack, while maintaining a zero-valued pitch rate. For a unit step change in pitch rate, the grid rotates at a constant, unit pitch rate. Because angle-of-attack changes with rotation, an additional grid motion is specified to aft and upward to eliminate angle-of-attack contributions. This grid motion technique can also be applied to lateral motions allowing the approximation of indicial responses with respect to sideslip angle, roll rate, and yaw rate. The benefit of this approach is that indicial responses, for $2 \mathrm{D}$ or $3 \mathrm{D}$ configurations, can be calculated at any flight condition, overcoming the identification problems experienced by previous methods. These indicial responses may then be used in Eqs. 2 and 3 to predict the unsteady aerodynamic response of a vehicle to any arbitrary trajectory. 

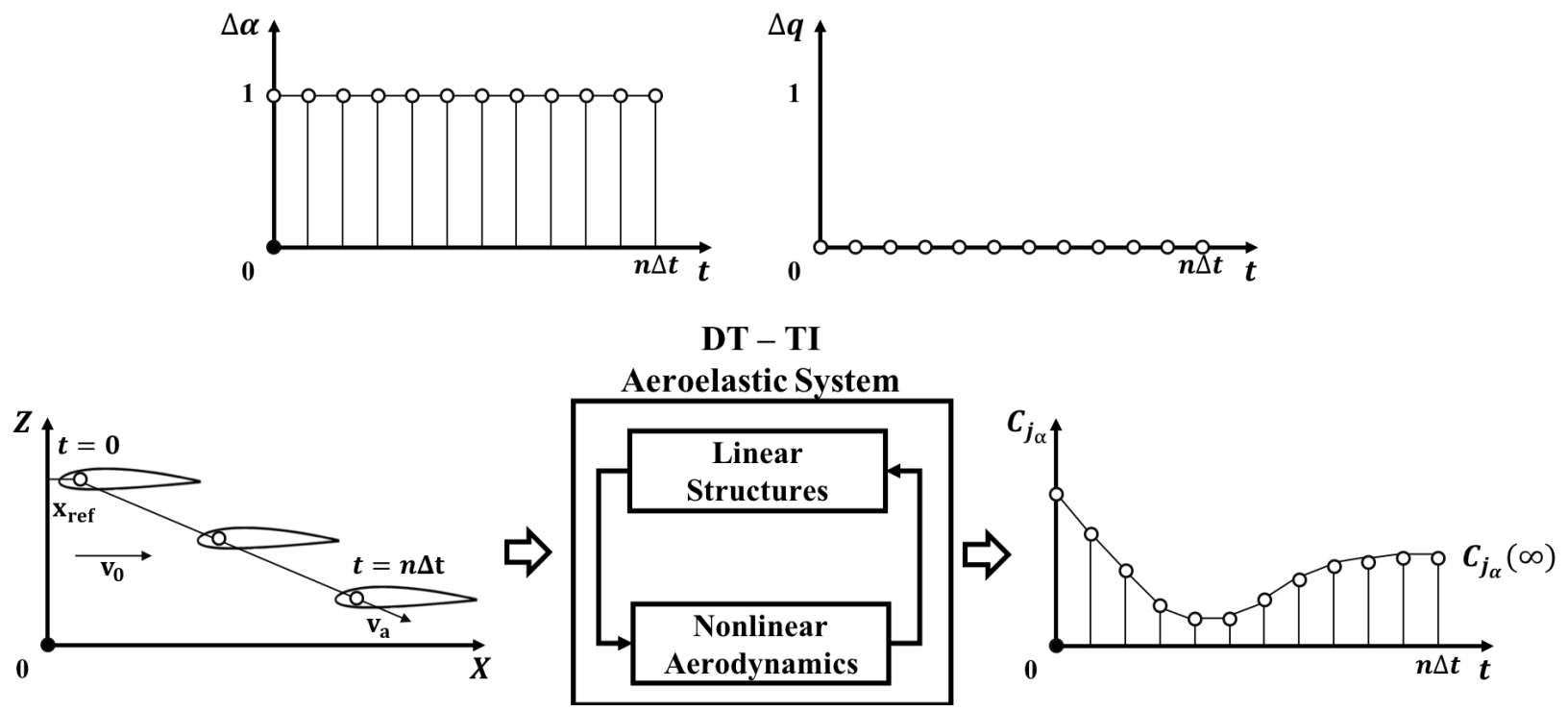

Figure 8. CFD simulation of angle-of-attack indicial responses.
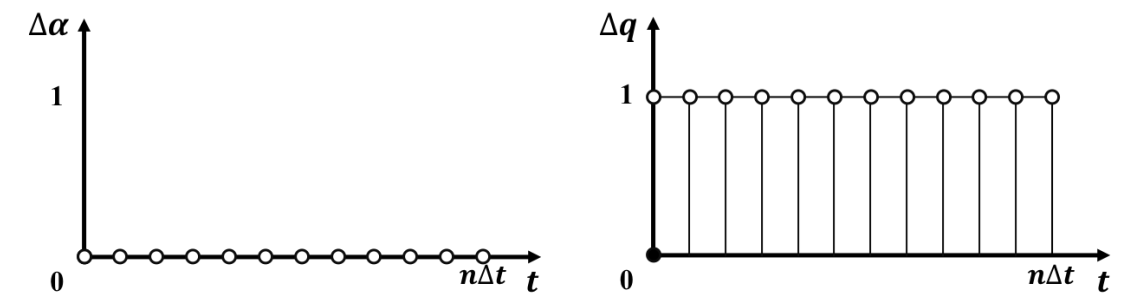

DT - TI

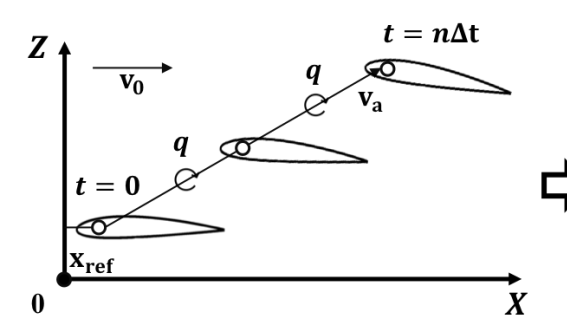

Aeroelastic System

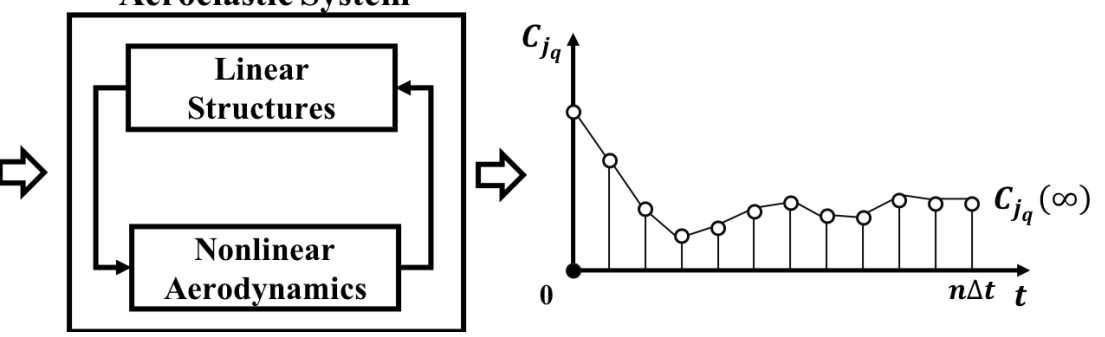

Figure 9. CFD simulation of pitch rate indicial responses.

The dynamic aeroelastic simulation used in the present work is governed by coupling the Euler equations with a linear structural modal solver. Each set of equations represents a discrete-time, time-invariant system, which may be described by indicial response theory provided the equations are well-converged in time. There are two requirements for simulating aeroelastic indicial responses: (1) a small enough time step be chosen to adequately resolve the highest frequency structural mode used to model the vehicle structure, and (2) a sufficient number of time steps are prescribed to attain a converged solution. The identified aeroelastic indicial responses are then capable of capturing the complete frequency spectrum of fluid-structure interactions present in a flexible vehicle's response to changes in the time-varying vehicle-state parameters.

For linear flow regimes, these aeroelastic indicial responses may be used in Eqs. 2 and 3 to predict the unsteady aeroelastic response to arbitrary trajectories defined in terms of $\alpha, \beta, p, q$ and $r$. Since the model assumes a linear relationship between the aerodynamic response and the system inputs, e.g., angle-of-attack, the linear aeroelastic ROM is generally applicable to linear portions of the flight envelope and limited to small-amplitude motions (locally linear) in nonlinear flight regimes, such as prestall flight. In the present work, linear forced oscillations will be evaluated using a 
linear aeroelastic indicial response ROM, Eqs. 2 and 3, which requires only a single indicial response with respect to each vehicle-state parameter at the initial flight condition.

For nonlinear flows, the linear aerodynamic indicial response theory of Eqs. 2 and 3 may be extended by use of parameterized indicial response functions [30,31]. Analagous to aerodynamic databases, flow nonlinearity may be captured by sampling a system's indicial response as a function of the flight space parameter values, where the degree of nonlinearity resolved scales with the sampling of indicial responses. The generalized nonlinear indicial response model for longitudinal and lateral coefficients are given by Eqs. 4 and 5 , respectively. Previous studies [20, 32, 33] implementing nonlinear indicial response ROMs for rigid vehicles typically assume a nonlinear dependency on angle-of-attack and Mach number for the indicial responses with respect to angle-of-attack $\left(C_{j_{\alpha}}\right)$ and sideslip angle $\left(C_{k_{\beta}}\right)$, while the indicial responses with respect to rotation rates $\left(C_{j_{q}}, C_{k_{p}}, C_{k_{r}}\right)$ are assumed to vary only with Mach number.

$$
\begin{gathered}
C_{j}(t)=C_{j_{0}}(M)+\frac{d}{d t}\left[\int_{0}^{t} C_{j_{\alpha}}(t-\tau, \alpha, M) \alpha(\tau) d \tau\right]+\frac{d}{d t}\left[\int_{0}^{t} C_{j_{q}}(t-\tau, M) q(\tau) d \tau\right] \\
C_{k}(t)=\frac{d}{d t}\left[\int_{0}^{t} C_{k_{\beta}}(t-\tau, \alpha, M) \beta(\tau) d \tau\right]+\frac{d}{d t}\left[\int_{0}^{t} C_{k_{p}}(t-\tau, M) p(\tau) d \tau\right]+\frac{d}{d t}\left[\int_{0}^{t} C_{k_{r}}(t-\tau, M) r(\tau) d \tau\right]
\end{gathered}
$$

In order to calculate the nonlinear responses, $C_{j \alpha}(t, \alpha, M)$ for example, the flight maneuver space is first parameterized by a set of angle-of-attack samples, $\alpha=\left[\alpha_{1}, \alpha_{2}, \ldots, \alpha_{n}\right]$, and a set of Mach number samples, $M=\left[M_{1}, M_{2}, \ldots, M_{n}\right]$. The responses are then calculated at each angle-of-attack, $\alpha_{i}(i=1,2, \ldots, n)$, for each Mach number sample, $M_{i}$, by first converging a static aeroelastic simulation at the given angle-of-attack and Mach number and then performing a dynamic aeroelastic simulation, for which grid motion capabilities are used to perform a unit step in angle-of-attack to $\alpha=\alpha_{i}+1$. At each time step, the fluid-structure interactions are resolved through coupling between the flow and structural solvers. The response function is then computed from the CFD solution by taking the difference between the time-varying response due to the unit step change and the static initial solutions at $\alpha=\alpha_{i}$. This process is repeated for each unique combination of flight space sampling for a total number of indicial response simulations equal to $\alpha_{i} \times M_{i}$ for the nonlinear response $C_{j \alpha}(t, \alpha, M)$. These same simulation requirements apply to the nonlinear responses with respect to sideslip angle, $C_{k \beta}(t, \alpha, M)$. The angular rate indicial responses, $C_{j q}(t, M), C_{\kappa p}(t, M)$, and $C_{\kappa r}(t, M)$, require sampling only as a function of Mach number at the initial angle-of-attack.

The flight space sampling may be completed using a variety of strategies, including the methods of full-factorial design, Latin hypercube sampling, or sampling based on optimality criteria. Indicial response samples for angle-of-attack and Mach number should be chosen to define the limits of the flight space corresponding to the flight maneuvers to be evaluated by the reduced-order models. The density of sampling should be carefully selected as to balance the computational requirements with the nonlinearity resolution needed for the maneuvers to be predicted.

Historically, in application to rigid vehicles, only positive angle-of-attack values have been sampled, as angle-ofattack indicial responses $\left(C_{j \alpha}\right)$ are assumed to be symmetric at negative angles of attack. This assumption is generally valid for small-magnitude, negative angles of attack, but can introduce modeling inaccuracies for larger negative values of angle-of-attack. For flexible vehicles, this sensitivity is stronger, and the applicability of this modeling assumption becomes limited to smaller angles of attack. Finally, it is typically assumed for rigid vehicles that the aerodynamic response for positive and negative steps in angle-of-attack are approximately the same. However, for flexible vehicles, the vehicle structure is not usually designed for symmetric load profiles. For this reason, negative steps may be necessary for longitudinal aeroelastic indicial responses as a means of capturing this asymmetric response observed in full-order simulations. In the present work, nonlinear forced pitch oscillations will be evaluated using Eq. 4 without including the dependency on freestream Mach number (constant). For this model, angle-of-attack indicial responses are simulated at 1 degree increments of angle-of-attack over the range covered by the pitching amplitude. 


\section{Results \& Discussion}

The applicability of the aeroelastic indicial ROM is explored through the simulation of the X-56A aircraft undergoing a series of forced harmonic oscillations at baseline cruise conditions. Prior to model evaluation, a static characterization study is conducted to identify the linear, nonlinear, and unsteady portions of the flight envelope. Characterization of these flight regimes allows for a more judicious selection of test maneuvers for linear and nonlinear ROM evaluations. Rigid and aeroelastic indicial responses are simulated (and compared) for ROM generation. Modeling accuracy will be evaluated by comparing with the full CFD flight maneuver rigid and aeroelastic solutions. Cost savings of the ROM will be assessed for both rigid and aeroelastic simulations to understand the scaled benefits of multidisciplinary unsteady aerodynamic ROMs.

\section{A. Static Characterization}

Prior to simulating dynamic maneuvers and developing reduced-order models, it is necessary to characterize the nonlinear and unsteady aerodynamic regimes of the X-56A. By identifying the unsteady aerodynamic regimes, test maneuvers may be defined to avoid near-stall flight conditions. Identification of flight envelope nonlinearity allows for the linear and nonlinear ROMs to be evaluated within their corresponding limits of application. It is important to reemphasize that 'nonlinearity' in the present work refers to the relationship between aerodynamic coefficients and vehicle-state parameters. Additionally, rigid and aeroelastic simulations are compared to observe how these regimes may vary when accounting for vehicle flexibility.

Toward this purpose, a series of static rigid and static aeroelastic time-accurate Euler flow solutions were generated at the baseline flight conditions across a range of $\alpha=\left[-5^{\circ}\right.$ to $\left.20^{\circ}\right]$. Per Eq. 6, a physical time step of $\Delta t=0.0004 \mathrm{~s}$ was chosen, where $f_{A W 1 T}$ is the highest frequency structural mode, i.e., assymetric wing first torsion, and $N \cong 200$.

$$
\Delta t=\frac{1}{f_{A W 1 T} N}
$$

Defining the time step in terms of the highest frequency structural mode ensures that any potential aeroelastic instabilities across the frequency spectrum may be resolved. A total of 10,000 time steps were simulated at each angle-of-attack to guarantee convergence at lower angles of attack and to resolve unsteady aerodynamic phenomena at higher angles of attack. Aeroelastic simulations were restarted from the converged rigid solutions to aid in numerical stability at higher angles of attack.

Figure 10 illustrates the static lift and pitching moment coefficient results for both the rigid (blue) and flexible (red) vehicle. Each data point represents the average aerodynamic coefficient value over the last 1,000 time steps of the time-accurate simulations. Additionally, the minimum and maximum coefficient values over the last 1,000 time steps are represented at each angle-of-attack by error bars, which serve as a measure of flow unsteadiness. The choice of 1,000 time steps was adequate for capturing several cycles of solution periodicity at the higher angles of attack.

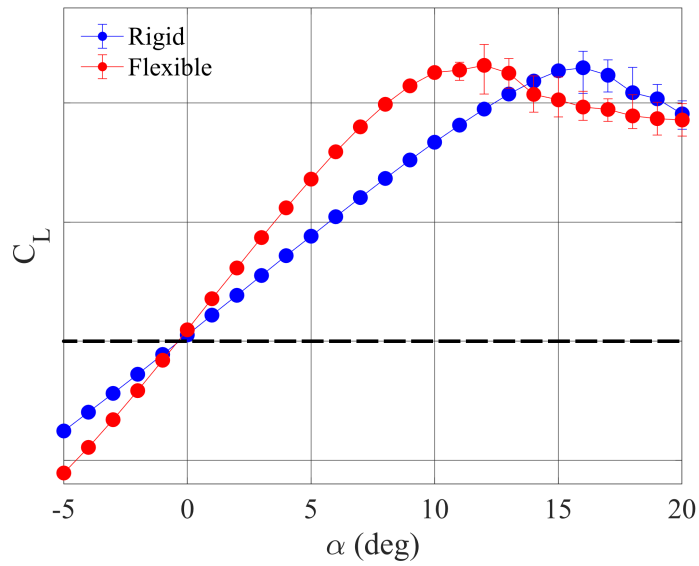

(a) Lift coefficient

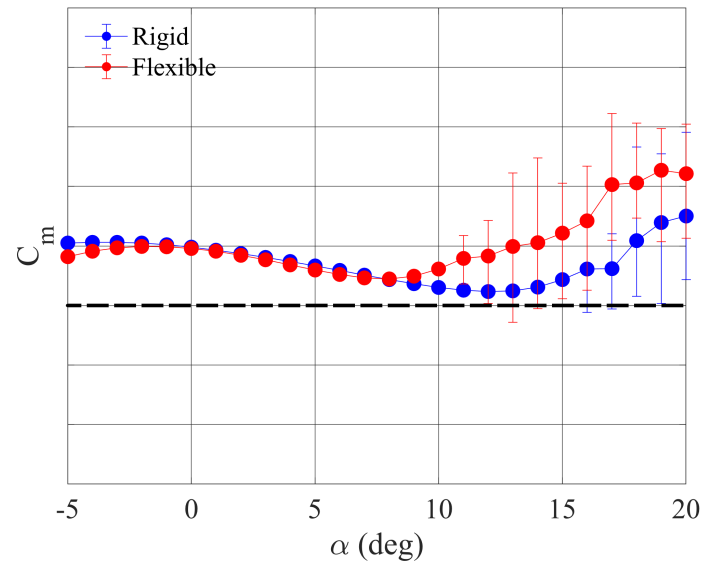

(b) Pitching moment coefficient

Figure 10. X-56A static lift and pitching moment coefficient for rigid (blue) and flexible (red) vehicle structure. 
The aerodynamics of the rigid vehicle is characterized by a nonlinear stall pitch-up behavior at higher angles of attack with unsteady effects beginning near $\alpha=16^{\circ}$ as a result of periodic vortex shedding. For $\alpha<12^{\circ}$, the aerodynamic response follows a generally linear trend with no significant measure of flow unsteadiness. A gradual pitching moment break is observed near $\alpha=12^{\circ}$. In comparison, the flexible vehicle exhibits greater nonlinearity and unsteadiness across the entire range of angle-of-attack.

At $\alpha=0^{\circ}$, the aerodynamic coefficient results for the rigid and flexible vehicle are fairly similar. For angles of attack up to $\alpha=13^{\circ}$, the flexible vehicle experiences a significant increase in lift coefficient relative to the rigid vehicle with the largest difference occuring at $\alpha=10^{\circ}$, where the lift coefficient is approximately $45 \%$ greater. Additionally, the presence of unsteady flow phenomena is observed beginning at $\alpha=11^{\circ}$, much earlier than the rigid vehicle at $\alpha=16^{\circ}$. For $\alpha>12^{\circ}$, the lift coefficient begins to decrease more gradually before reaching a value comparable to that of the rigid vehicle. The pitching moment remains comparable up to $\alpha=8^{\circ}$ before increasing at a greater rate relative to the rigid vehicle, which is indicative of earlier flow separation on the wing. Mesh boundary animations were generated at the converged flight conditions to provide further insight into how the structure deforms subjects to fluid-structure interactions.

Figure 11] shows the static deformation of the flexible vehicle (red) compared to the rigid vehicle (blue) for $\alpha=10^{\circ}$ at the baseline flight condition. The front and side views depict a noticeable wingtip deflection, in addition to a pitch-up deformation of the vehicle's center-body, which provides rationale for the observed increase in lift coefficient. A three-view illustrated in Fig. 11(c) also shows a small reverse twist of the winglet and a slight decrease in wing sweep. The increased deflection and higher incidence angle at the wingtip would explain the observation of an earlier onset stall accompanied by stronger unsteady aerodynamic phenomena. The results further demonstrate the pronounced flexibility of the X-56A aeroservoelasticity technology testbed aircraft.

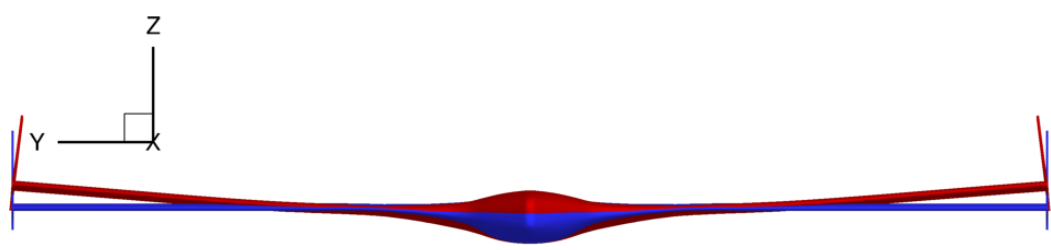

(a) Front view

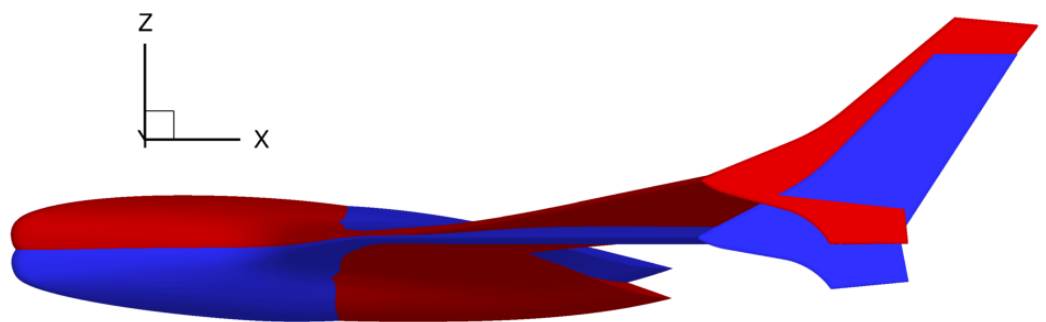

(b) Side view

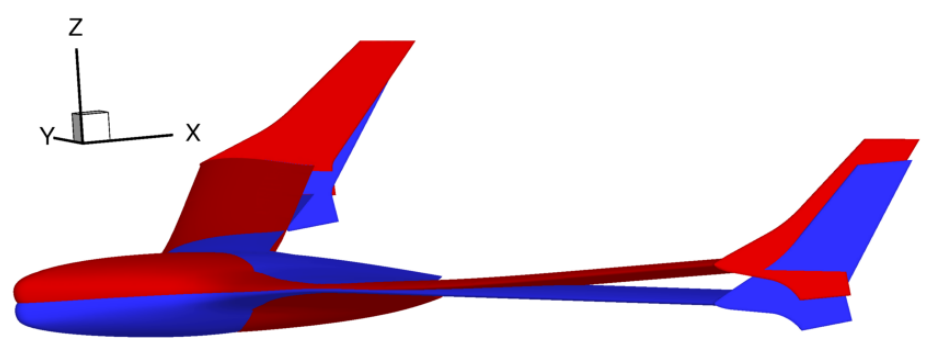

(c) Three-view

Figure 11. Static deformation of the flexible vehicle (red) compared to the rigid vehicle (blue) at $\alpha=1^{\circ}$, $\mathbf{M}=\mathbf{0 . 1 3}$. 
Overall, the static aerodynamic characterization illustrates a significant difference in the extent of linear and nonlinear angle-of-attack ranges between the rigid and flexible X-56A aircraft. The aerodynamic response of the rigid vehicle remains fairly linear for $\alpha=\left[0^{\circ}\right.$ to $\left.10^{\circ}\right]$ with a stall region beginning at $\alpha=16^{\circ}$, as commonly observed for subsonic vehicles. In contrast, the flexible vehicle shows a generally nonlinear aerodynamic response even at lower angles of attack with locally linear variations confined to amplitudes of $\Delta \alpha=\left[1^{\circ}\right.$ to $\left.3^{\circ}\right]$, and an unsteady aerodynamic region beginning at $\alpha=11^{\circ}$. The impact of a deforming center-body and wing results in an overall higher lift coefficient and a more limited flight envelope when avoiding the presence of unsteady aerodynamic phenomena. Because the X-56A was designed as a testbed aircraft for aeroservoelastic technology, its significant flexibility promotes an increase in aerodynamics loading, as opposed to the damping generally observed for performance vehicles. By characterizing the flight envelope, a more judicious selection of flight maneuvers may be made in evaluating the linear and nonlinear variants of the aeroelastic ROM.

\section{B. Description of Test Maneuvers}

The salient scaling parameter for the forced oscillation simulations is reduced frequency $(k)$, given in terms of motion frequency or angular frequency by Eq. 7 . In this expression, reduced frequency is defined in terms of the motion frequency $(f)$, a reference length $\left(L_{r e f}\right)$, and the freestream velocity $\left(U_{\infty}\right)$. For the test cases shown in Table 2 , the reference length is equal to the semichord length $\left(c_{r e f} / 2\right)$ for pitch oscillations and the semispan length $\left(b_{r e f} / 2\right)$ for roll and yaw oscillations.

$$
k=\frac{2 \pi f L_{r e f}}{U_{\infty}}
$$

Simulated reduced frequencies correspond to varying levels of aerodynamic unsteadiness and are generally classified by the following [34]:

- Quasisteady $-0 \leq k \leq 0.05$

- Unsteady - $0.05 \leq k \leq 0.2$

- Highly unsteady $-k>0.2$

Table 2 lists the forced oscillation test cases to be simulated by the linear and aeroelastic indicial response ROMs. Each of the test cases are simulated at a constant Mach number of $M=0.13$, coinciding with the X-56A aircraft cruise condition. For the linear ROM evaluations, a mean angle-of-attack of $\alpha_{0}=0^{\circ}$ is used for forced roll, yaw, and pitch oscillations. An oscillation amplitude of $\Delta=1^{\circ}$ is selected for each oscillation type to ensure a locally linear response. To evaluate the nonlinear ROM as a function of angle-of-attack, forced pitching oscillations are simulated at a mean angle-of-attack of $\alpha_{0}=5^{\circ}$ with a pitching amplitude of $\alpha_{A}=4^{\circ}$. The result is a forced pitch oscillation transversing $\alpha=\left[1^{\circ}\right.$ to $\left.9^{\circ}\right]$ to establish the limitations of the linear ROM and the benefits of a nonlinear ROM.

Table 2. X-56A forced harmonic oscillation test cases.

\begin{tabular}{|c|c|c|c|c|}
\hline ROM Applicability & Oscillation Type & $\alpha_{0}(\mathrm{deg})$ & Amplitude $(\mathrm{deg})$ & $\mathrm{k}$ \\
\hline \hline Linear & Roll/Yaw/Pitch & 0 & 1 & 0.05 \\
\hline Linear & Roll/Yaw/Pitch & 0 & 1 & 0.10 \\
\hline Linear & Roll/Yaw/Pitch & 0 & 1 & 0.20 \\
\hline Nonlinear & Pitch & 5 & 4 & 0.05 \\
\hline Nonlinear & Pitch & 5 & 4 & 0.10 \\
\hline Nonlinear & Pitch & 5 & 4 & 0.20 \\
\hline
\end{tabular}

For each of the forced oscillation types, the oscillations are simulated at reduced frequencies of $k=0.05,0.10$, and 0.20. By evaluating each maneuver for increasingly unsteady flows, the ability of the aeroelastic indicial responses to capture the unsteady aerodynamics of flexible flight vehicles over a wide frequency spectrum can be assessed. Additionally, this provides an opportunity to compare how the incorporation of flexibility effects changes the unsteady aerodynamic response for the X-56A aircraft undergoing forced oscillations, and how these changes differ for various rates of motion. 


\section{Generation of Indicial Response Solutions}

\section{Solution Strategy}

The indicial responses are simulated by imposing through rigid-body grid motion a sudden step change in $\alpha, \beta$, or angular rates from a converged static solution at each initial angle-of-attack in Table 3 . The FUN3D grid-motion files are created by mapping the trajectory vehicle-state inputs in Table 4, to the transformations noted of Ref. [20]. The incremental steps are defined as $\Delta \alpha=1^{\circ}, \Delta \beta=1^{\circ}$, and $\Delta p=\Delta q=\Delta r=1 \mathrm{rad} / \mathrm{s}=180 / \pi \mathrm{deg} / \mathrm{s}$. For an $\alpha$-step, the initial time step is set to $\alpha=\alpha_{0}, \beta=0^{\circ}$, and $\phi=\theta=\psi=0^{\circ}$. This produces a pure translation motion downward and slightly aft to maintain $\Delta q=0 \mathrm{rad} / \mathrm{s}$. The $\beta$-, $p$-, $q$-, and $r$ - steps are initialized by setting $\alpha=\beta=\phi=\theta=\psi=0^{\circ}$ for the initial time step. For a $\beta$-step, this imposes a pure translation to the right and slightly aft to maintain $\Delta r=0 \mathrm{rad} / \mathrm{s}$. For a $p$-step, this results in an impulsive roll of the grid about the body axis. For a $q$-step, a constant-rate pitch rotation of the grid coupled with an upward and aft translation along a curved path that maintains $\alpha=\beta=0^{\circ}$ throughout the motion. For a $\Delta r$-step, there is a constant-rate yaw rotation of the grid coupled with a sideways and aft translation, such that $\alpha=\beta=0^{\circ}$ is maintained during the motion.

Table 3. Matrix of indicial step cases for ROM identification.

\begin{tabular}{cc}
\hline \hline$\alpha_{0}(\mathrm{deg})$ & $M=0.13$ \\
\hline 0 & $\Delta \alpha, \Delta \beta, \Delta p, \Delta q, \Delta r$ \\
1 to 9 & $\Delta \alpha$ \\
\hline \hline
\end{tabular}

Table 4. Trajectory parameters for creating indicial response trajectories $(t>0)$.

\begin{tabular}{ccccccc}
\hline \hline Indicial & $\alpha(\operatorname{deg})$ & $\beta(\operatorname{deg})$ & $\phi(\mathrm{deg})$ & $\theta(\mathrm{deg})$ & $\psi(\mathrm{deg})$ & $M_{0}$ \\
\hline$\alpha$ - step & $\alpha_{0}+1$ & 0 & 0 & 0 & 0 & 0.13 \\
$\beta$ - step & 0 & 1 & 0 & 0 & 0 & 0.13 \\
$p$ - step & 0 & 0 & $\Delta p \cdot t$ & 0 & 0 & 0.13 \\
$q$ - step & 0 & 0 & 0 & $\Delta q \cdot t$ & 0 & 0.13 \\
$r$ - step & 0 & 0 & 0 & 0 & $\Delta r \cdot t$ & 0.13 \\
\hline \hline
\end{tabular}

Indicial response simulations require a well-converged static initial solution. An efficient initialization approach uses the maximum allowable time step to converge the static aeroelastic solution using the minimum number of iterations. For the static characterizations, a physical time step of $\Delta t=0.0004 \mathrm{~s}$ was chosen based on resolving the highest frequency structural mode by a sampling factor of $N \approx 200$. While appropriate for dynamic aeroelastic simulations, this time step value is ineffecient for steady flight regimes. Additionally, the static aeroelastic solutions were restarted from a well-converged static solution for the rigid vehicle. This practice is generally advisable for improving numerical stability for flexible vehicle simulations at rigorous flight conditions, but unnecessary for the X-56A flight envelope. Efficient static time-accurate aeroelastic initialization solutions were generated using a time step of $\Delta t=0.5 \mathrm{~s}$ based on resolving a single period of the highest frequency structural mode. With this resolution, well-converged static aeroelastic solutions were obtained using 75 time steps. The same solution strategy was used for both rigid and aeroelastic initializations. The indicial step responses were simulated in FUN3D using the temporal parameters presented in Table 5 A total of 4,000 time steps were simulated based on resolving five periods of the lowest frequency structural mode. This ensures that the fluid-structure interactions are resolved for each indicial response simulation. The same solution strategy was used for rigid indicial responses for consistent comparisons.

Table 5. Temporal parameters for indicial response simulations (rigid and flexible).

\begin{tabular}{cccccc}
\hline \hline Mach & $\Delta t(\mathrm{~s})$ & $s$ & $N_{\text {sub }}$ & Total Time Steps & Total Iterations \\
\hline 0.13 & $4.0 \mathrm{E}-4$ & 0.002 & 20 & 4,000 & 80,000 \\
\hline \hline
\end{tabular}




\section{Indicial Response Solutions}

Figure 12 illustrates the lift and pitching moment coefficient indicial responses with respect to step changes in angle-of-attack and pitch rate for the flexible and rigid vehicle simulations at zero angle-of-attack. The response values are plotted versus a nondimensional time, $s=V t / c_{r e f}$, which represents the distance traveled by a fluid particle in terms of integer semichord lengths. Each of the lift coefficient responses for the rigid vehicle exhibit initial transients as the flow adjusts to the impulsive unit change in angle-of-attack before converging at the $\alpha=\alpha_{i}+1^{\circ}$ state near $s=40$. In contrast, the aeroelastic responses exhibit a longer duration and larger magnitude initial transient followed by a damping of fluid-structure interactions until the solution converges near $s=250$. Similar behavior is observed for the pitching moment coefficient responses. It is important to note that because the aeroelastic indicial responses require approximately five times the number of time steps for convergence relative to the rigid indicial responses, the influence of previous motion states is likely to be more pronounced for full-order simulations.

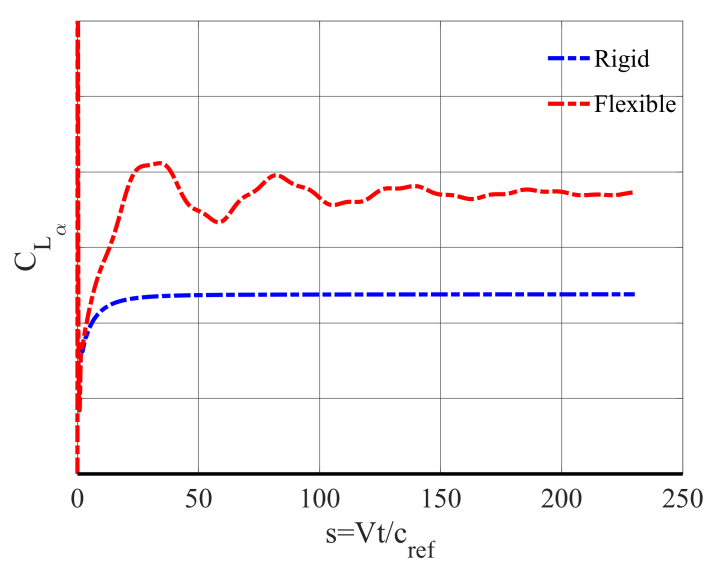

(a) Lift coefficient - $\alpha$-step

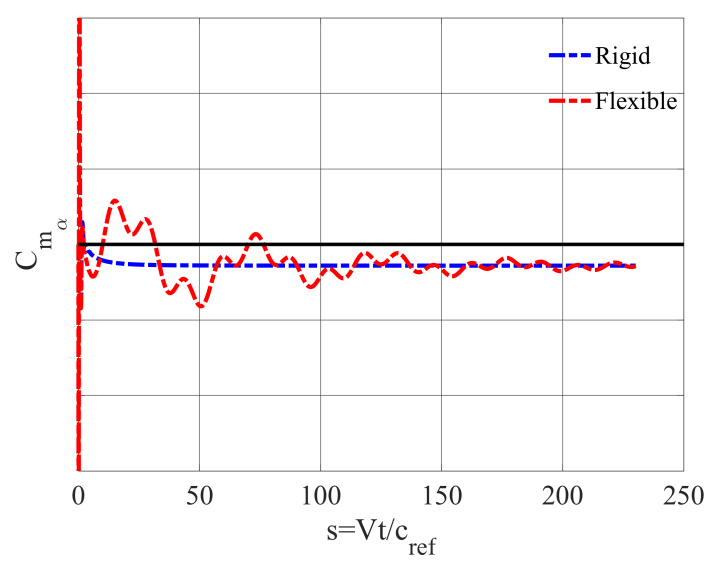

(c) Pitching moment coefficient - $\alpha$-step

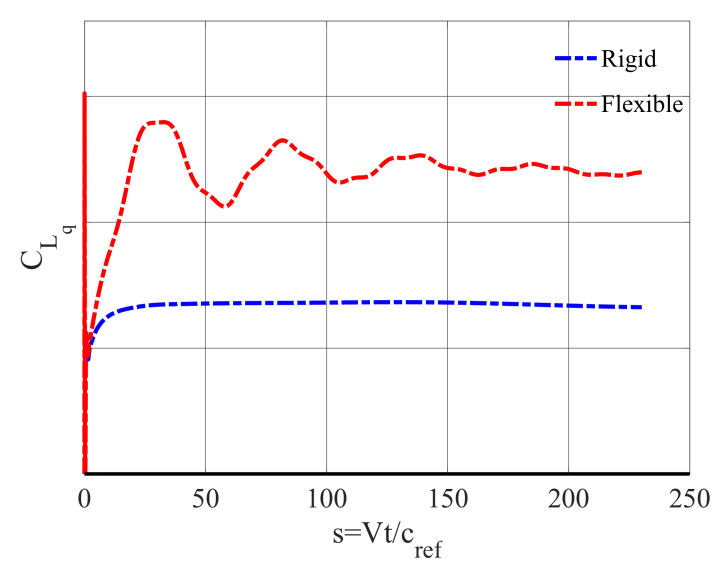

(b) Lift coefficient - $q$-step

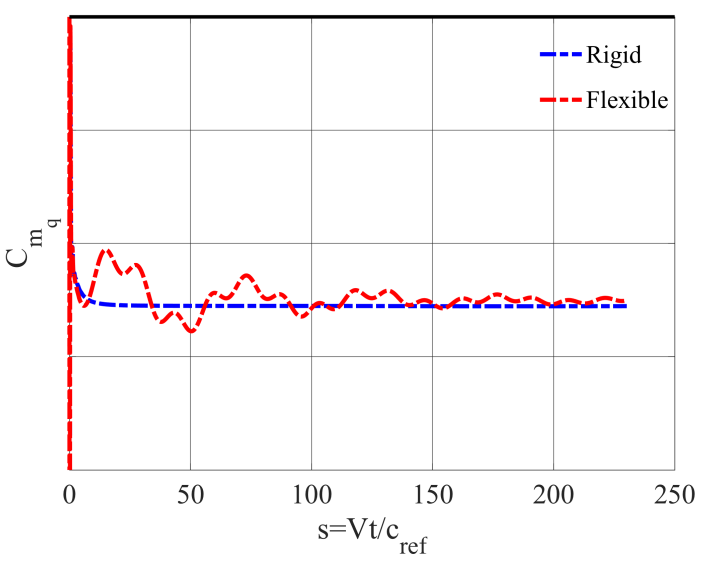

(d) Pitching moment coefficient - $q$-step

Figure 12. Lift (top) and pitching moment (bottom) coefficient indicial responses with respect to unit steps in angle-of-attack (left) and pitch rate (right) for the flexible (red) and rigid (blue) vehicles at $\alpha=0^{\circ}$. 
Figure 13 illustrates the side force, rolling moment, and yawing moment coefficient indicial responses with respect to step changes in roll rate and yaw rate for the flexible and rigid vehicle simulations at zero angle-of-attack. For both the $p$-step and $r$-step, the side force response is characterized by an initial transient peak due to the initial grid rotation, where a converged response is attained by $s=25$. The differences in these responses due to vehicle flexibility are negligible. This is generally expected as the coupled fluid-structure interactions are strongest in the longitudinal plane, where the structure is more prone to deformation due to aerodynamic loading. Similar dynamic behavior is shown for the rolling moment coefficient response with respect to a step change in yaw rate. For a step change in roll rate, periodic oscillations are observed in the aeroelastic response near the rigid response mean value. The yawing moment coefficient responses for each step type show negligible differences for the rigid and flexible vehicle. Figure 14 illustrates the side force, rolling moment, and yawing moment coefficient indicial responses with respect to step changes in sideslip angle for the flexible and rigid vehicle simulations at zero angle-of-attack. Similar to the step in roll rate, the rolling moment coefficient indicial response illustrates the most pronounced differences relative to the rigid vehicle with periodic oscillations centered around a slightly larger converged derivative value.

For nonlinear ROM evaluations, the indicial responses with respect to a step change in angle-of-attack were sampled as a function of angle-of-attack. The lift and pitching moment coefficient responses for the rigid (blue) and aeroelastic (red) simulations are shown in Fig. 15 Each of the lift coefficient responses for the rigid vehicle exhibit initial transients as the flow adjusts to the impulsive unit change in angle-of-attack before converging at the $\alpha=\alpha_{i}+1^{\circ}$ state near $s=40$. The responses initialized from $\alpha=0^{\circ}$ and $5^{\circ}$ are nearly identical, confirming the linearity of the flight envelope within the given angle-of-attack range, as previously shown in Fig. 10. The response at $\alpha=9^{\circ}$ exhibits convergence to a lower response value than the lower angles of attack. This result confirms the smaller lift curve slope and the need to consider a nonlinear model for the rigid X-56A aircraft maneuvering beyond $\alpha>8^{\circ}$. Pitching moment coefficient response results illustrate a less significant variation with an increase in angle-of-attack, and in fact, no discernable trend as the response at $\alpha=5^{\circ}$ has the most negative value. This observation is in agreement with the static characterization shown in Fig. 10 , where the pitching moment varies linearly up to $\alpha=10^{\circ}$ with slight variations in slope within each two degree interval.

In comparison, the aeroelastic responses illustrate a greater nonlinearity with significant differences evident even between the lower angle-of-attack responses at $\alpha=0^{\circ}$ and $5^{\circ}$. The response at $\alpha=9^{\circ}$ converges to a smaller mean value, resulting in a strong nonlinearity as previously shown in Fig. 10. The large variability in the mean response values suggests that an increased sampling of aeroelastic indicial responses with respect to angle-of-attack will be required for resolving maneuvers within a fixed amplitude range, relative to the rigid response sampling. Additionally, the responses exhibit a periodic, yet stable unsteadiness as a result of fluid-structure interactions. These interactions appear to be of smaller magnitude and higher frequency with an increase in angle-of-attack.

In contrast to the rigid responses, the pitching moment coefficients for the aeroelastic responses present a nonlinear variation within the sampled angle-of-attack range. Moreover, the response at $\alpha=9^{\circ}$ has a positive mean value, whereas the lower angle-of-attack responses have a negative mean value. This result confirms the observations made from the static characterization plots of Fig. 10, where a pitching moment break occurs earlier for the flexible vehicle at $\alpha=8^{\circ}$ compared to the rigid vehicle at $\alpha=13^{\circ}$. A periodic oscillation is also observed for the pitching moment coefficient, though similar in frequency and magnitude with change in angle-of-attack.

Overall, the angle-of-attack indicial response sensitivity to angle-of-attack directly correlates with the nonlinearity of the static and static aeroelastic characterization curves. Thus, a nonlinear dependence on angle-of-attack should be considered when predicting longitudinal coefficients for maneuvers outside the ranges of $\alpha=\left[0^{\circ}\right.$ to $\left.5^{\circ}\right]$ for the rigid vehicle and maneuvers for which $\Delta \alpha \geq 1^{\circ}$ anywhere in the flight envelope for the flexible vehicle. Additionally, these initial aeroelastic step responses appear to capture complex fluid-structure interactions, which periodically oscillate at a given flight condition. This result suggests that hysteresis effects will be of greater importance for flexible vehicles, and the aeroelastic ROM may provide increased performance benefits compared to a traditional quasisteady stability derivative model than a rigid $\mathrm{ROM}$ 


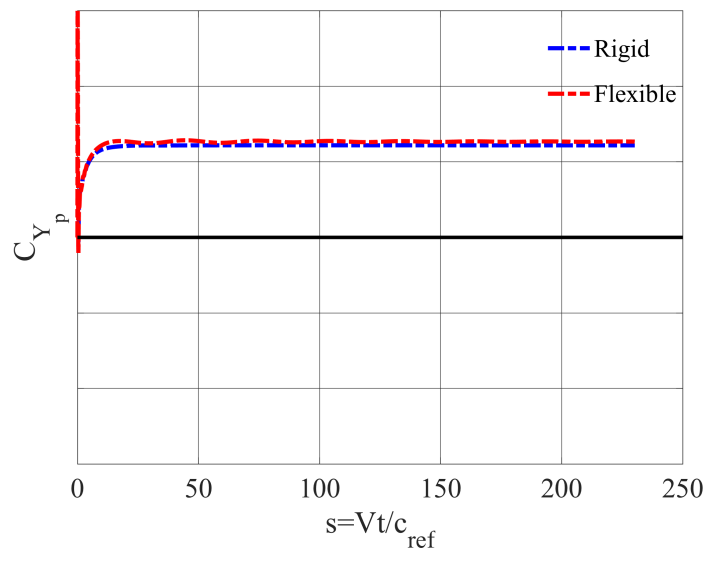

(a) Side force coefficient - $p$-step

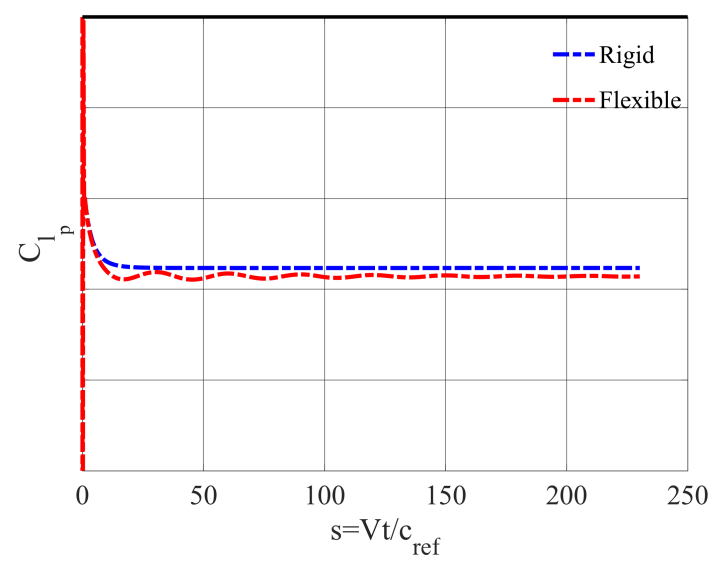

(c) Rolling moment coefficient - $p$-step

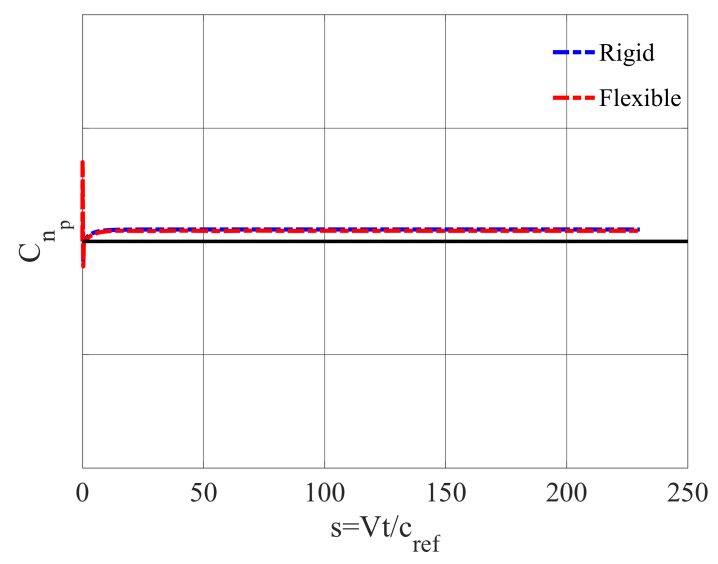

(e) Yawing moment coefficient - $p$-step

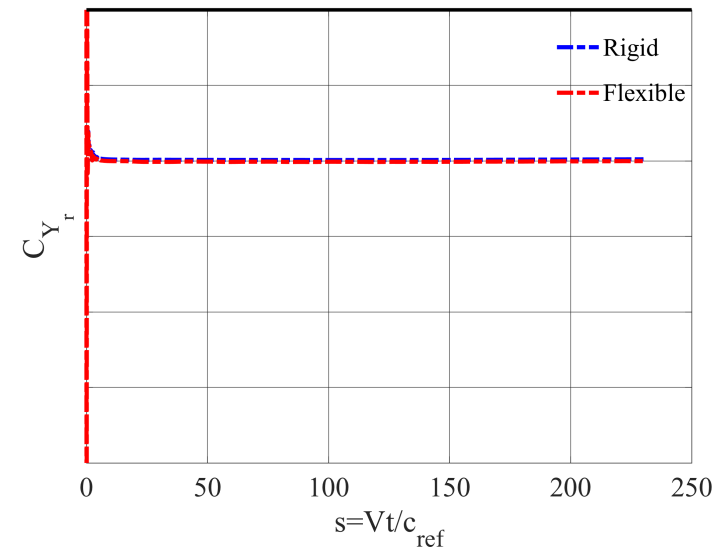

(b) Side force coefficient - $r$-step

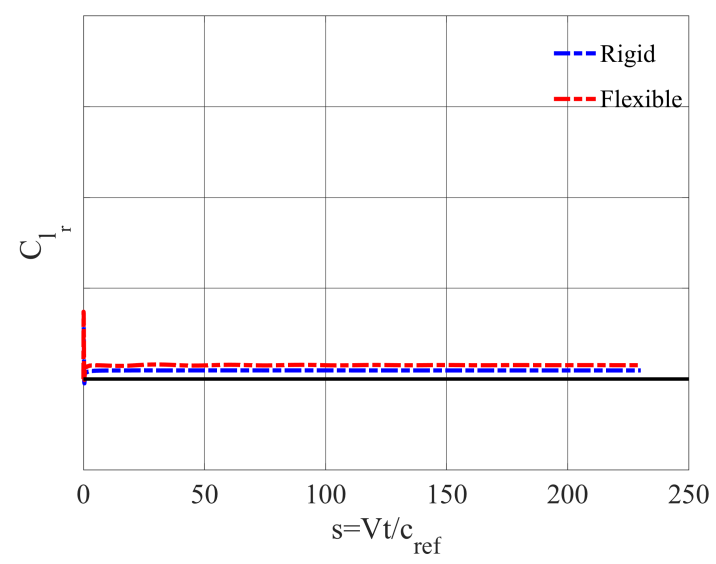

(d) Rolling moment coefficient - $r$-step

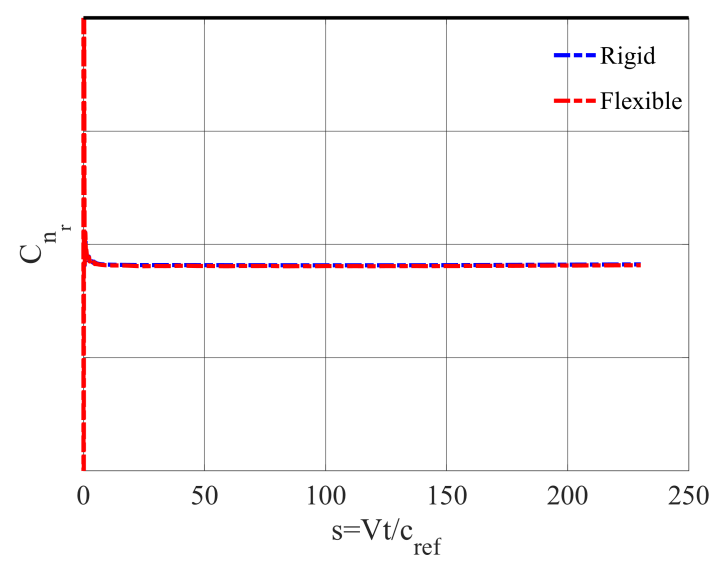

(f) Yawing moment coefficient - $r$-step

Figure 13. Side force (top), rolling moment (middle), and yawing moment (bottom) coefficient indicial responses with respect to unit steps in roll rate (left) and yaw rate (right) for the flexible (red) and rigid (blue) vehicle at $\alpha=\mathbf{0}^{\circ}$. 


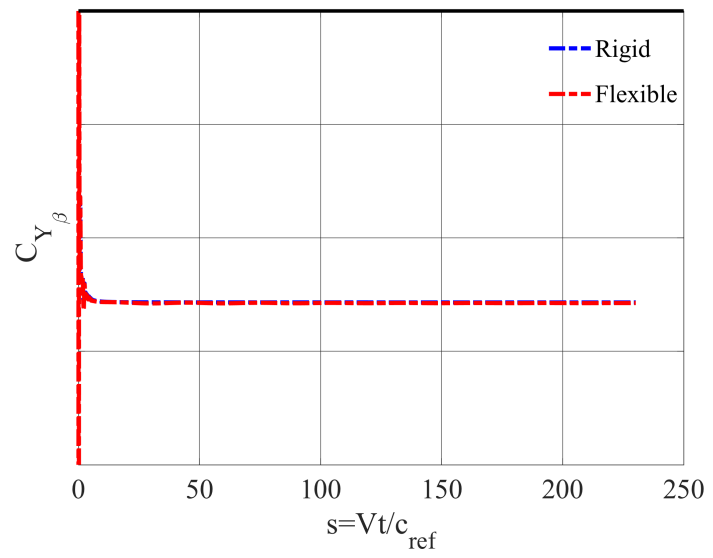

(a) Side force coefficient $-\beta$-step

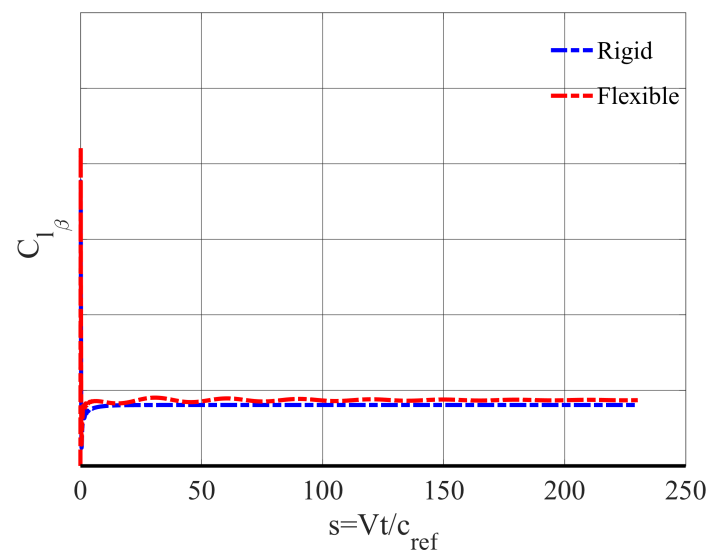

(b) Rolling moment coefficient - $\beta$-step

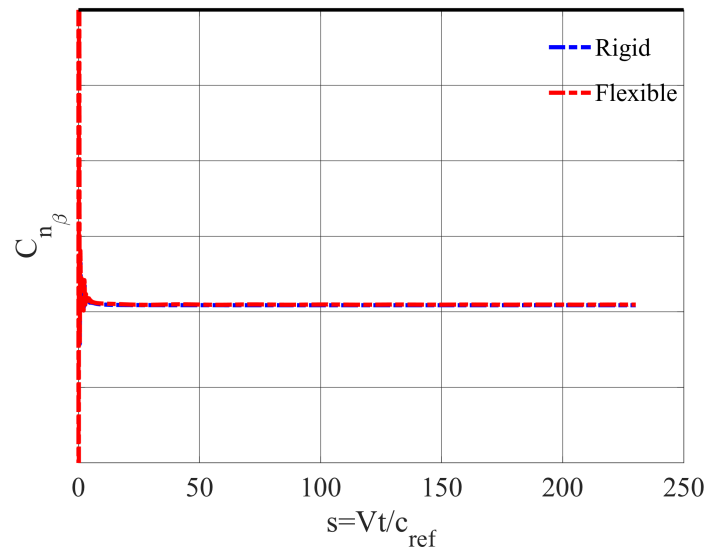

(c) Yawing moment coefficient - $\beta$-step

Figure 14. Side force (top), rolling moment (middle), and yawing moment (bottom) coefficient indicial responses with respect to a unit step in sideslip angle for the flexible (red) and rigid (blue) vehicle at $\alpha=0^{\circ}$. 


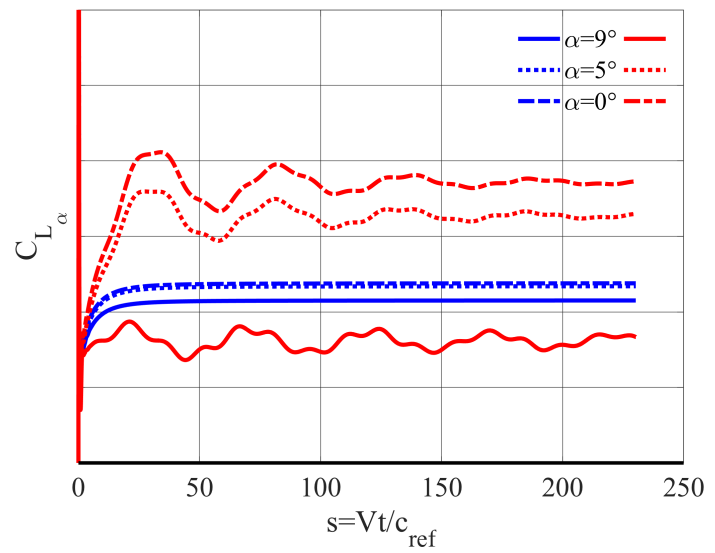

(a) Lift coefficient - $\alpha$-step

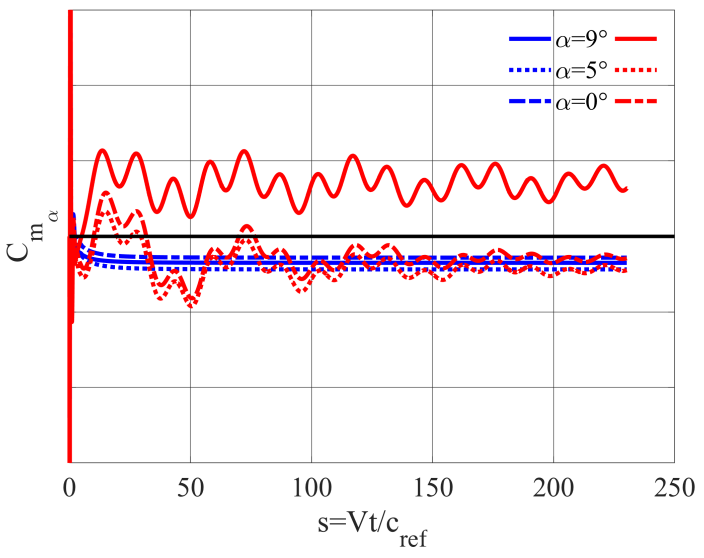

(b) Pitching moment coefficient - $\alpha$-step

Figure 15. Lift (left) and pitching moment (right) coefficient indicial responses with respect to a unit step in angle-of-attack for the flexible (red) and rigid (blue) vehicle at $\alpha=0^{\circ}, 5^{\circ}$, and $9^{\circ}$.

\section{Evaluation of Reduced-Order Model}

Each forced oscillation case was initialized from a well-converged static time-accurate simulation. For the aeroelastic simulations, a static aeroelastic time-accurate simulation was conducted without using a static time-accurate restart solution. Both the rigid and aeroelastic simulations were advanced in time using a physical time step of $\Delta t \approx 0.5$ for 75 time steps, where 20 subiterations were used to converge the solution in pseudotime. The static initialization time step is approximately the same as the period of the highest frequency structural mode and provides an efficient means of producing a converged static aeroelastic response for the forced oscillation initial condition. The solution strategy implemented was consistent for the rigid and aeroelastic forced oscillation cases and was chosen to satisfy the more strict temporal resolution requirements for the flexible vehicle. To elaborate, the most dynamic motion case $(k=0.20)$ corresponds to an oscillation frequency of $4.58 \mathrm{~Hz}$, whereas the highest structural mode frequency is much greater at $11.95 \mathrm{~Hz}$. Thus, a time step selected based on resolution of the much greater structural mode frequency will be more than sufficient in simulating dynamic motions of any feasible frequency of interest. A sampling factor of $N=200$ was selected to resolve the highest frequency structural mode, resulting in a normalized time step of $s \approx 0.002$ for all dynamic simulations. This is consistent with the dynamic solution strategy implemented for indicial responses and will be used for all forced oscillation simulations, providing a well-converged dynamic solution and allowing for a one-to-one comparison for computational costs. Three cycles were simulated for both the rigid and flexible vehicle cases to allow for the initial transient response to decay and to explore potential differences in the convergence of the hysteresis loops when accounting for structural flexibility.

\section{Forced Rolling Oscillations}

Forced rolling oscillations were prescribed starting from an initial roll angle of $\phi=0^{\circ}$ with a roll amplitude of $\Delta \phi=1^{\circ}$ at the baseline flight condition of $\alpha=0^{\circ}, \beta=0^{\circ}$, and $M=0.13$. The amplitude was limited to one degree to ensure a locally linear aerodynamic response with respect to the roll angle. The motion was simulated at reduced frequencies of $k=0.05,0.10$, and 0.20 . This increase in reduced frequency translates to a decrease in maneuver time, which serves to illicit a more unsteady aerodynamic response where the influences of previous motion states are more prominent.

Figure 16 illustrates a comparison between CFD and ROM side force coefficient results for each of the forced rolling oscillation cases (top to bottom) for the rigid (left) and flexible (right) vehicle cases. Figure 17 shows the rolling moment coefficient results. The side force coefficient solution is characterized by an initial, positive peak due to the onset of grid motion followed by a transient decay in the first 0.5 degree change in rolling amplitude leading to a clockwise hysteresis loop. In contrast, the rolling moment coefficient is characterized by an initial, negative peak followed by a quicker transient degree in the first 0.25 degree change in rolling amplitude leading to a counterclockwise hysteresis loop. The impact of increasing the reduced frequency is an aerodynamic damping effect, observed by a widening of the hysteresis 
loops for both coefficients. For each successive increase in reduced frequency, a proportional widening of the loops is observed. The minimum and maximum side force and rolling moment values are not affected by reduced frequency. Overall, nearly identical results are observed between the rigid and flexible simulations. While difficult to see, the only notable difference observed is a longer duration transient decay, where the transient decay for each coefficient persists as a small-magnitude periodic oscillation before converging to the hysteresis loop just before reaching peak positive amplitude within the first cycle. Such a result was to be expected based on the subtle differences between the rigid and aeroelastic indicial responses with respect to step changes in roll rate. In each case, the linear indicial response ROM shows an ability to accurately predict lateral unsteady aerodynamic coefficients for forced rolling oscillations under a wide range of reduced frequencies for both rigid and flexible simulations.

\section{Forced Yawing Oscillations}

Forced yawing oscillations were prescribed starting from an initial yaw angle of $\phi=0^{\circ}$ with a roll amplitude of $\Delta \psi=1^{\circ}$ at the baseline flight condition of $\alpha=0^{\circ}, \beta=0^{\circ}$, and $M=0.13$. The amplitude was limited to one degree to ensure a locally linear aerodynamic response with respect to the yaw angle. The motion was simulated at reduced frequencies of $k=0.05,0.10$, and 0.20 to observe the unsteady aerodynamic response variations due to different levels of dynamic motion.

Figure 18 illustrates a comparison between CFD and ROM side force coefficient results for each of the forced yawing oscillation cases (top to bottom) for the rigid (left) and flexible (right) vehicle cases. Figure 19 shows the yawing moment coefficient results. The side force coefficient solution is characterized by an initial, negative peak due to the onset of grid motion followed by an abrupt transient decay leading to a counterclockwise hysteresis loop. A similar trend is observed with respect to the yawing moment coefficient. The impact of increasing reduced frequency is an aerodynamic damping effect, observed by a thickening of the hysteresis loops for both coefficients. For each successive increase in reduced frequency, a proportional widening of the loops is observed. The minimum and maximum side force and yawing moment values are not affected by reduced frequency. Similar to the forced rolling oscillations, nearly identical results are observed between the rigid and flexible vehicles, which is expected due to the negligible difference between rigid and aeroelastic indicial responses. In each case, the linear indicial response ROM shows an ability to accurately predict lateral unsteady aerodynamic coefficients for forced yawing oscillations under a wide range of reduced frequencies for both rigid and flexible simulations.

\section{Forced Pitching Oscillations}

Two sets of forced oscillations were simulated to test the linear and nonlinear variants of the indicial response ROM. The linear forced pitching oscillations were prescribed starting from an initial angle-of-attack $\alpha_{0}=0^{\circ}$ with a pitch amplitude of $\Delta \theta=1^{\circ}$ at $\beta=0^{\circ}, M=0.13$. The amplitude was limited to 1 degree to ensure a locally linear aerodynamic response with respect to angle-of-attack. The nonlinear forced pitching oscillations were prescribed starting from an initial angle-of-attack $\alpha_{0}=5^{\circ}$ with a pitch amplitude of $\Delta \theta=4^{\circ}$ at $\beta=0^{\circ}, M=0.13$. The larger amplitude was chosen to transverse a greater portion of the flight envelope, extending into the nonlinear flight regime.

Figure 20 illustrates a comparison between CFD and ROM lift coefficient results for each of the linear forced pitching oscillation cases (top to bottom) for the rigid (left) and flexible (right) vehicle cases. Figure 21 shows the pitching moment coefficient results. For the rigid vehicle, the lift coefficient is characterized by an initial, positive peak due to the onset of grid motion followed by a transient decay that persists for the first quarter of the first cycle before converging to a clockwise hysteresis loop. The pitching moment coefficient encounters a strong negative peak, extending into the negative coefficient value range, followed by a more rapidly decaying transient response before converging to a counterclockwise hysteresis loop within the first eighth of the first cycle. The impact of increasing reduced frequency is an aerodynamic damping effect, observed by a widening of the hysteresis loops for both coefficients. Additionally, the minimum and maximum lift coefficient increase and decrease, respectively, as illustrated by a clockwise rotation of the hysteresis loop. By increasing the oscillation rate, the flow is unable to converge to a steady-state and the influence of previous motion states act to dampen the range of lift coefficient values encountered during oscillatory motions. The opposite trend is observed for the pitching moment coefficient with a counterclockwise rotation of the hysteresis loop.

The impact of fluid-structure interactions on the unsteady aerodynamic lift and pitching moment coefficient responses is significant. For each reduced frequency, the aerodynamic damping changes between successive cycles of motion with notable variations in hysteresis loop width, as well as the minimum and maximum coefficient values. Relative to the rigid simulations, the aerodynamic damping in the lift coefficient response is more significant for the $k=0.05$ and $k=0.10$ cases, as evidenced by wider hysteresis loops. Furthermore, the hysteresis loops are rotated counterclockwise, 


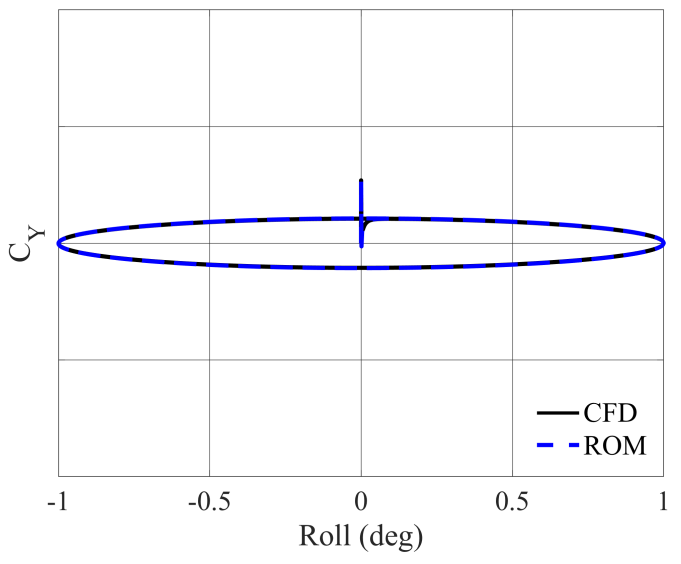

(a) Side force coefficient - Rigid $(k=0.05)$

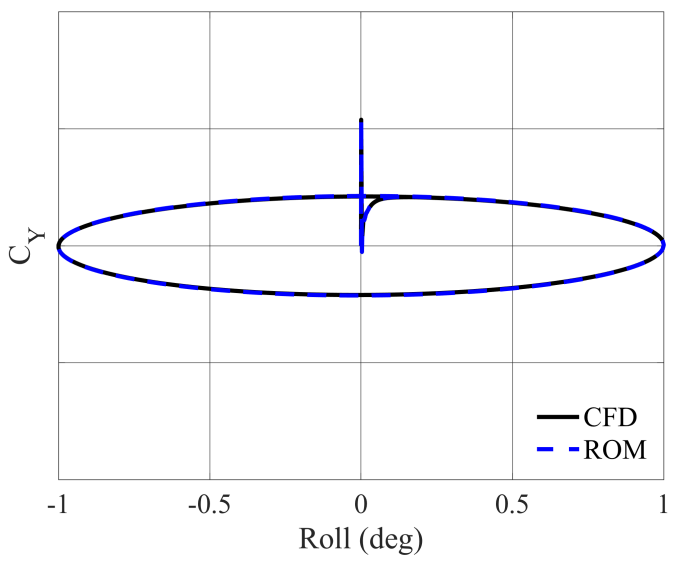

(c) Side force coefficient - Rigid $(k=0.10)$

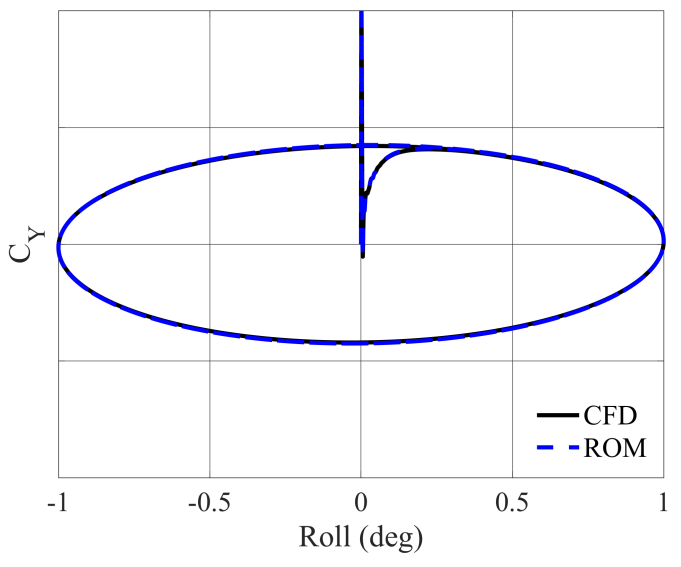

(e) Side force coefficient - Rigid $(k=0.20)$

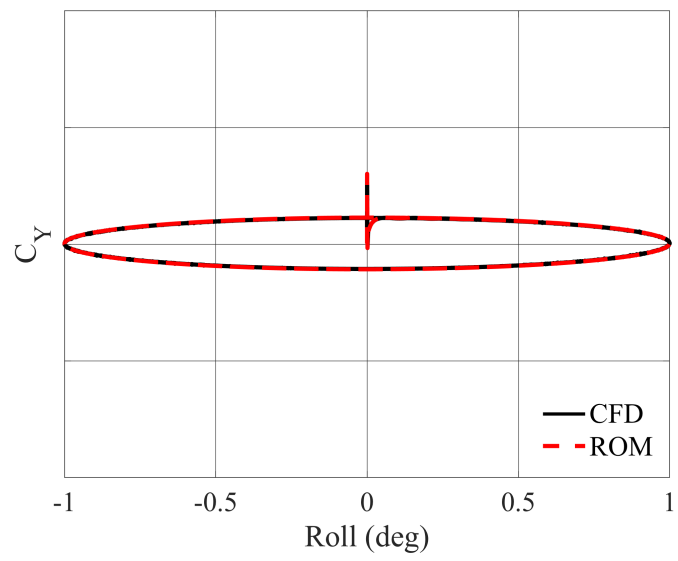

(b) Side force coefficient - Aeroelastic $(k=0.05)$

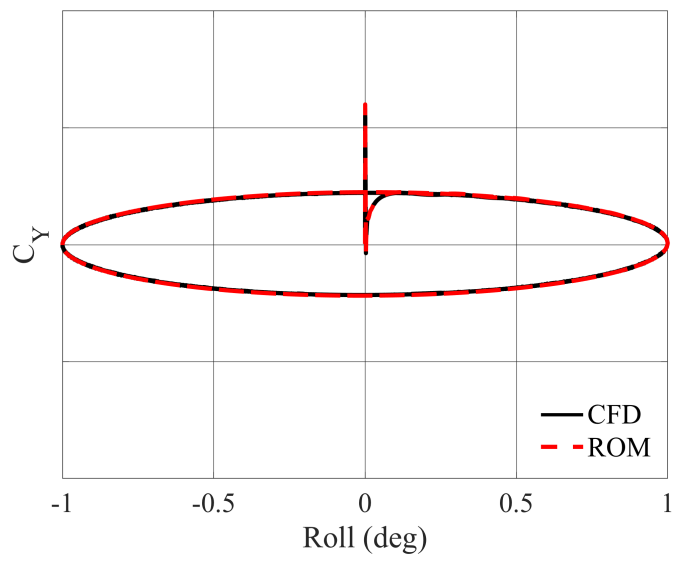

(d) Side force coefficient - Aeroelastic $(k=0.10)$

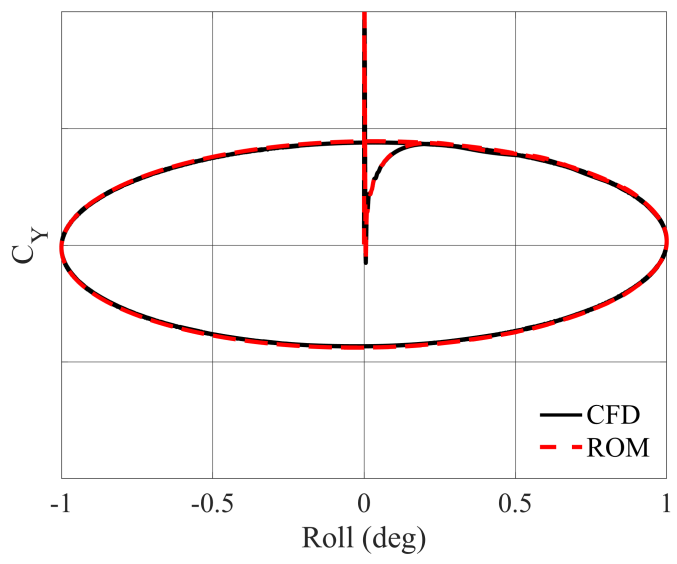

(f) Side force coefficient - Aeroelastic $(k=0.20)$

Figure 16. ROM vs. CFD side force coefficient results for the rigid (left) and flexible (right) X-56A performing a forced rolling oscillation with one degree amplitude for $\mathrm{k}=0.05$ (top), 0.10 (middle), and 0.20 (bottom) at $\alpha=\mathbf{0}^{\circ}, \beta=\mathbf{0}^{\circ}, \mathbf{M}=\mathbf{0 . 1 3}$. 


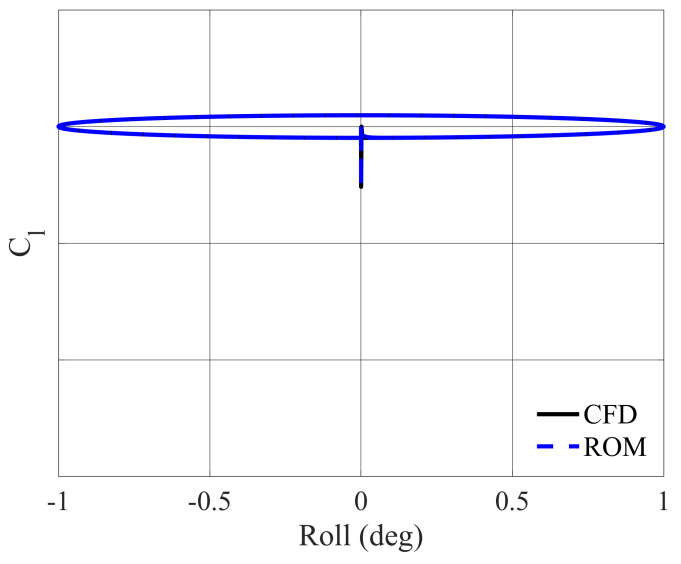

(a) Rolling moment coefficient - Rigid $(k=0.05)$

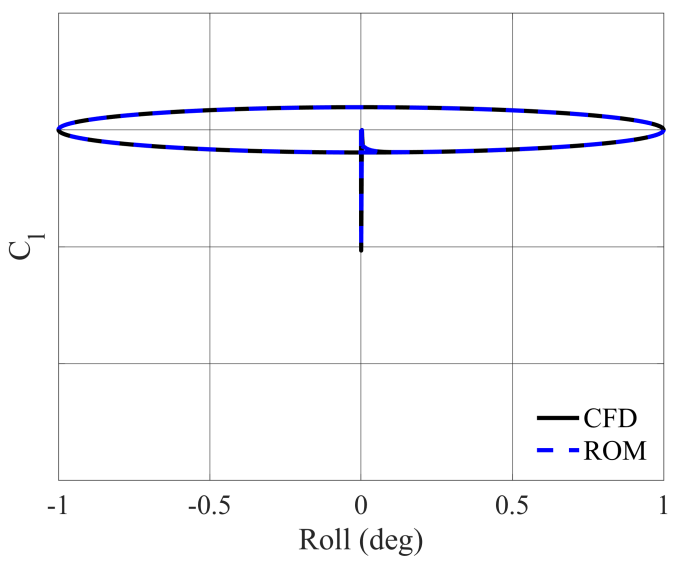

(c) Rolling moment coefficient - Rigid $(k=0.10)$

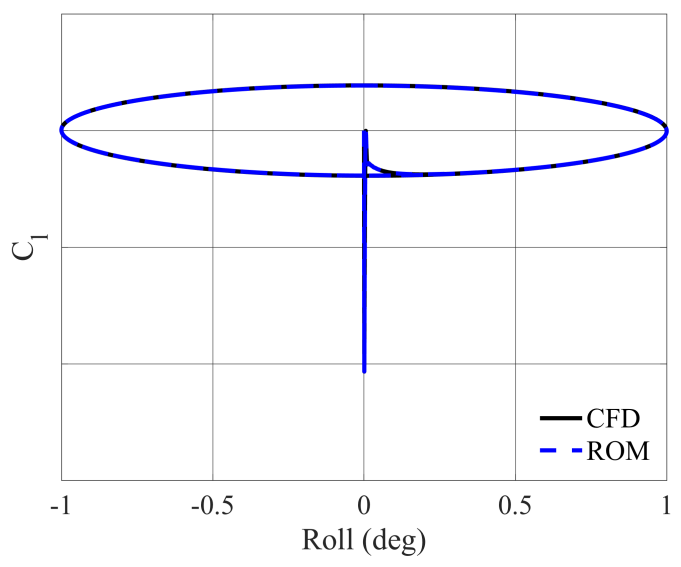

(e) Rolling moment coefficient - Rigid $(k=0.20)$

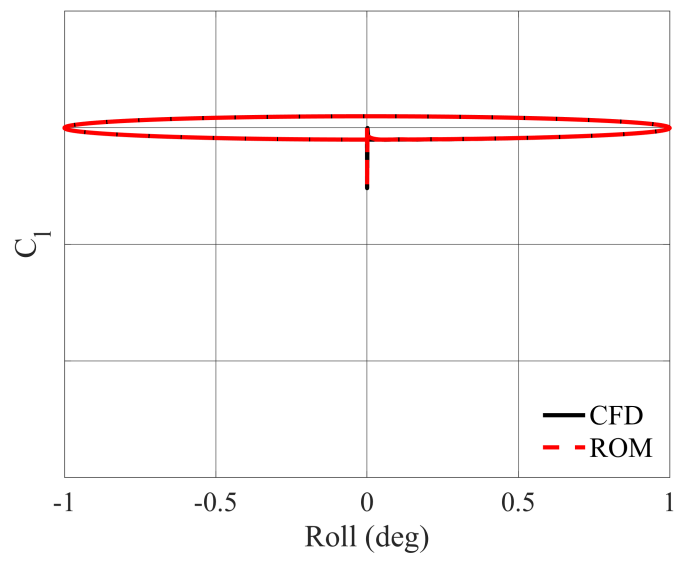

(b) Rolling moment coefficient - Aeroelastic $(k=0.05)$

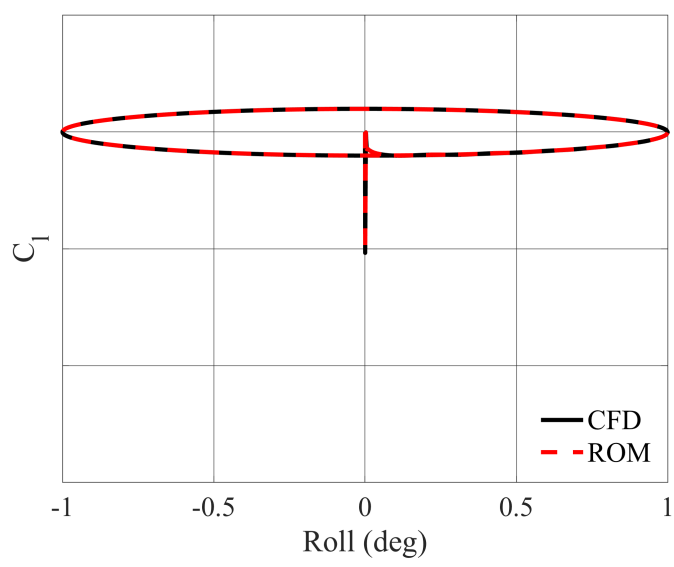

(d) Rolling moment coefficient - Aeroelastic $(k=0.10)$

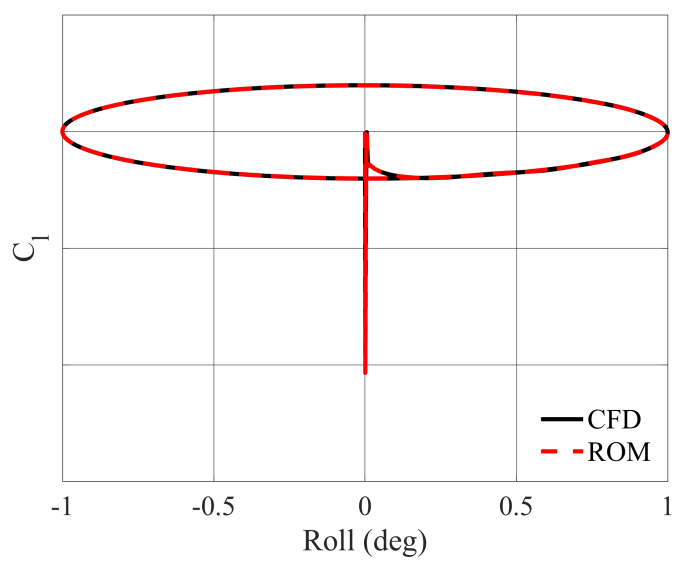

(f) Rolling moment coefficient - Aeroelastic $(k=0.20)$

Figure 17. ROM vs. CFD rolling moment coefficient results for the rigid (left) and flexible (right) X-56A performing a forced rolling oscillation with one degree amplitude for $k=0.05$ (top), 0.10 (middle), and 0.20 (bottom) at $\alpha=0^{\circ}, \beta=0^{\circ}, \mathbf{M}=\mathbf{0 . 1 3}$. 


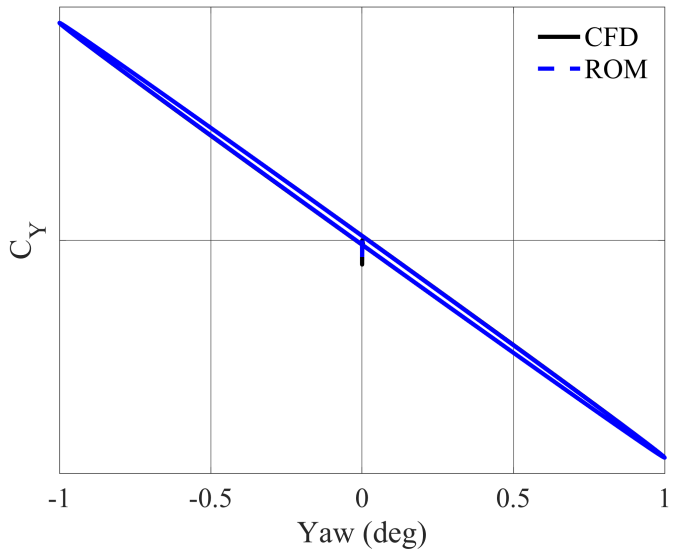

(a) Side force coefficient - Rigid $(k=0.05)$

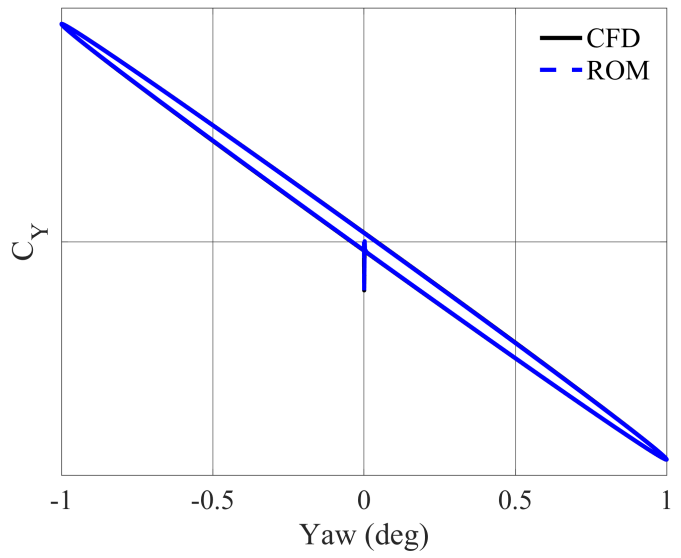

(c) Side force coefficient - Rigid ( $k=0.10)$

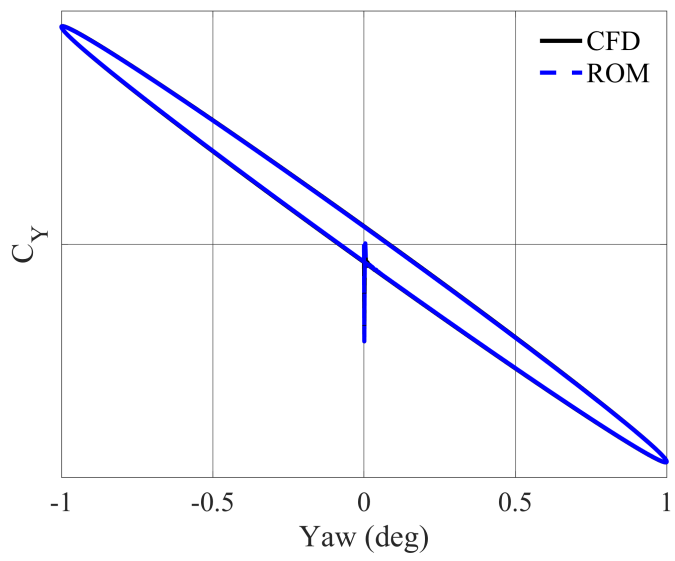

(e) Side force coefficient - Rigid $(k=0.20)$

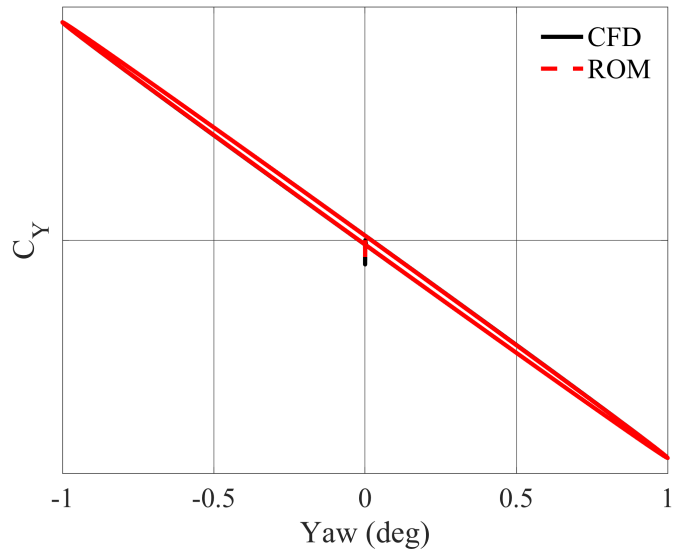

(b) Side force coefficient - Aeroelastic $(k=0.05)$

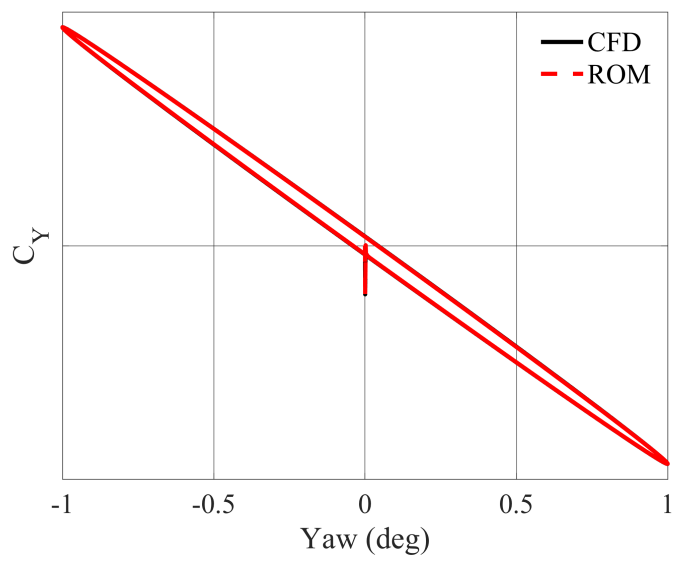

(d) Side force coefficient - Aeroelastic $(k=0.10)$

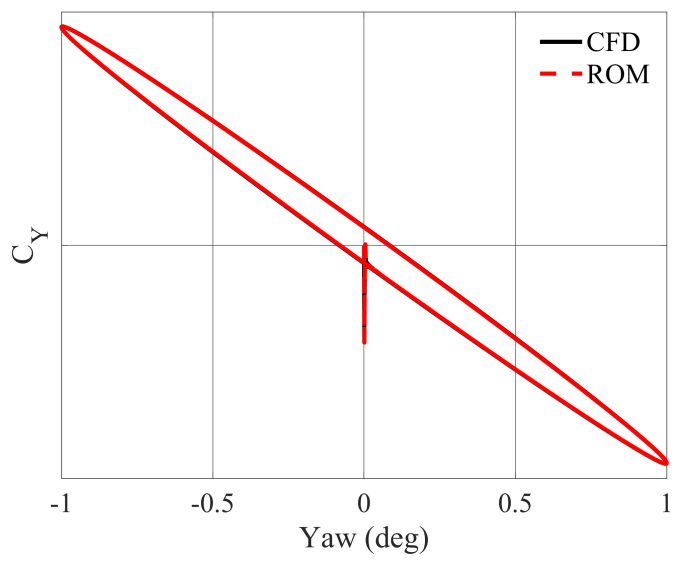

(f) Side force coefficient - Aeroelastic $(k=0.20)$

Figure 18. ROM vs. CFD side force coefficient results for the rigid (left) and flexible (right) X-56A performing a forced yawing oscillation with three degree amplitude for $\mathrm{k}=0.05$ (top), 0.10 (middle), and 0.20 (bottom) at $\alpha=\mathbf{0}^{\circ}, \beta=\mathbf{0}^{\circ}, \mathbf{M}=\mathbf{0 . 1 3}$. 


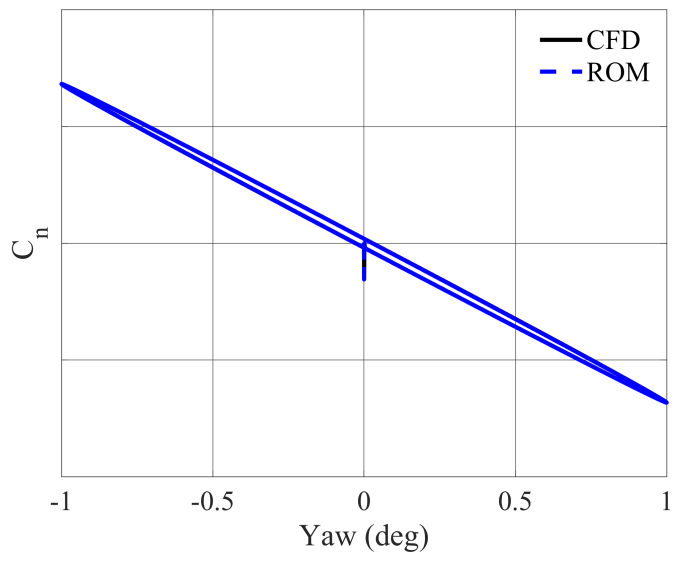

(a) Yawing moment coefficient - Rigid $(k=0.05)$

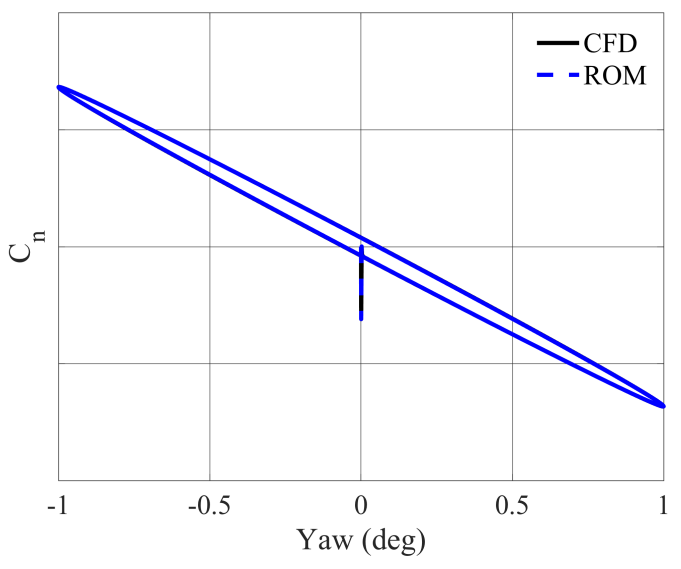

(c) Yawing moment coefficient - Rigid $(k=0.10)$

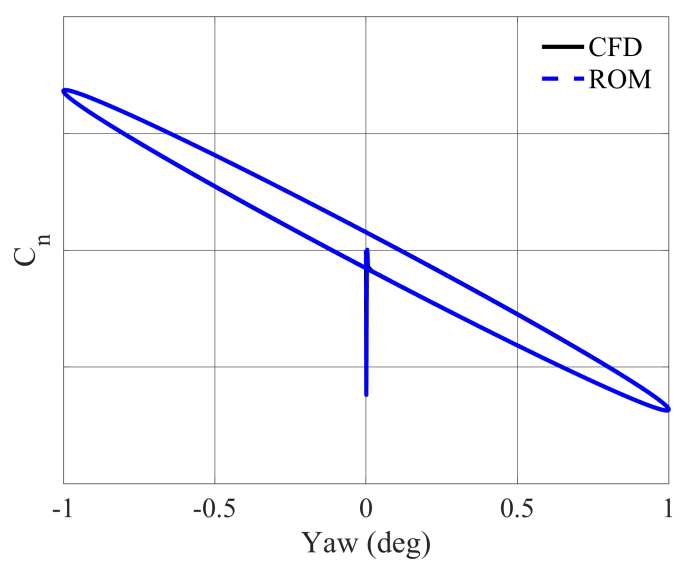

(e) Yawing moment coefficient - Rigid $(k=0.20)$

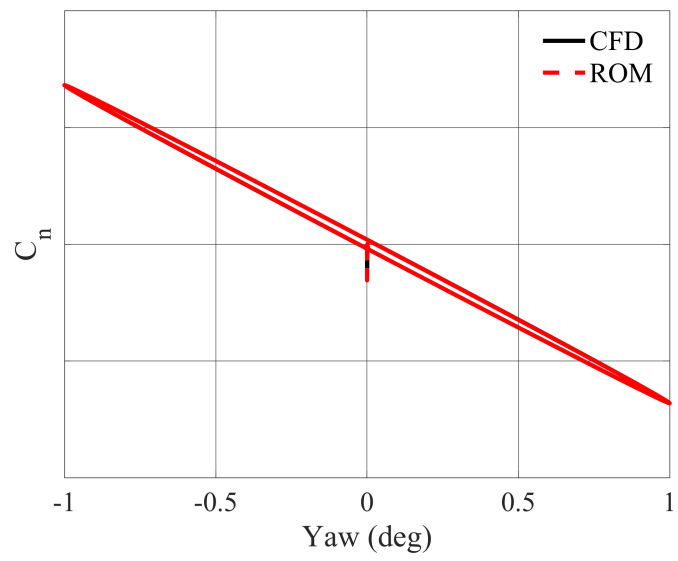

(b) Yawing moment coefficient - Aeroelastic $(k=0.05)$

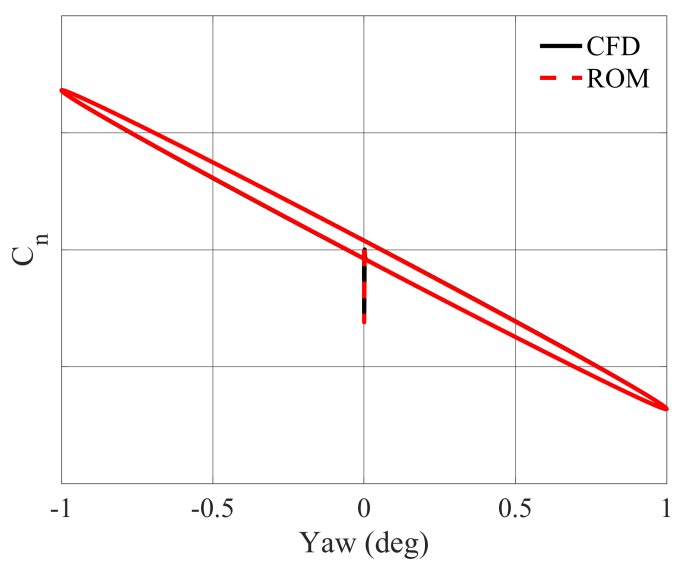

(d) Yawing moment coefficient - Aeroelastic $(k=0.10)$

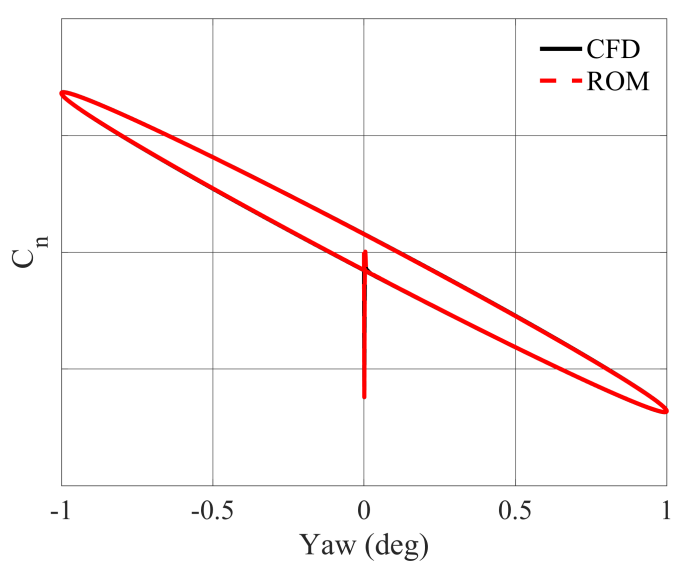

(f) Yawing moment coefficient - Aeroelastic $(k=0.20)$

Figure 19. ROM vs. CFD yawing moment coefficient results for the rigid (left) and flexible (right) X-56A performing a forced yawing oscillation with one degree amplitude for $k=0.05$ (top), 0.10 (middle), and 0.20 (bottom) at $\alpha=0^{\circ}, \beta=0^{\circ}, \mathbf{M}=\mathbf{0 . 1 3}$. 
translating to a wider range of lift coefficient values. In the $k=0.20$ case, the average aerodynamic damping is similar in magnitude compared to the rigid simulations, where the primary difference is the cycle-to-cycle variation due to fluid-structural interactions. It is interesting to note that an increase in the reduced frequency no longer corresponds to a straight-forward clockwise rotation of the hysteresis loops, as the $k=0.10$ case produces the largest variation in lift coefficient values.

Similar but less pronounced trends are found with respect to the pitching moment coefficient results. Relative to the rigid simulations, the variation in aerodynamic damping is less significant likely due to the pitching moment coefficient being smaller in magnitude. At the lower reduced frequencies, response nonlinearity is observed despite the limited motion amplitude. Such nonlinearities result from the influence of fluid-structure interactions, when the structural deformation is capable of converging toward static aeroelastic states for slowly-varying motions. Overall, the linear ROM predictions are in great qualitative agreement with CFD simulations, correctly resolving the initial transient response and the cycle-to-cycle differences in hysteresis loop widening/rotation.

Figure 22 illustrates a comparison between CFD and ROM lift coefficient results for each of the nonlinear forced pitching oscillation cases (top to bottom) for the rigid (left) and flexible (right) vehicle cases. Figure 23 shows the pitching moment coefficient results. The rigid lift and pitching moment coefficient results for the nonlinear pitch oscillation are qualitatively similar to the results for the low-amplitude pitch oscillation. This is to be expected given that the lift curve shown in Fig. 10 remains fairly linear over $\alpha=\left[1^{\circ}\right.$ to $\left.9^{\circ}\right]$. In contrast, the aeroelastic lift reponse shows a greater nonlinearity in the mean response values as the oscillation approaches peak amplitude values. This is particularly evident at the lower oscillation frequencies when the structure has time to deform further at a given angle-of-attack as shown by a decrease in aerodynamic damping and change in hysteresis slope near $\alpha=9^{\circ}$. Overall, the nonlinear ROM predictions show good qualitative agreement with CFD simulations over a wider range in angle-of-attack, correctly resolving the initial transient response and the hysteresis loop widening/rotation with increases in reduced frequency. The slight differences between the ROM and CFD results can be attributed to the linear interpolation used to model the responses, the finer sampling requirements for aeroelastic indicial responses, and/or the need to model differences in positive and negative steps for indicial response identification.

\section{E. Assessment of Reduced-Order Model Performance}

The computational resources required for creating the X-56A indicial response solutions and the 12 forced oscillation cases ( 3 frequencies for each type) are included in Table 6 . The FUN3D indicial solutions were computed on the NASA Langley K4-cluster, consisting of Intel Gold 6148 Skylake processors. Full-order maneuver solutions were computed on the NASA Advanced Supercomputing (NAS) facility's Electra supercomputer Skylake nodes, consisting of Intel Xeon Gold 6148 Skylake processors. Unsteady aeroelastic predictions for full-order maneuvers were generated using a CFD2ROM MATLAB code for indicial response modeling. The linear and nonlinear ROM predictions were computed in a matter of seconds for full-order maneuvers on a $2 \mathrm{GHz}$ Intel Core i7 MacBook Pro personal laptop. Due to the negligible online computation times, ROM computational costs are isolated to the costs incurred for indicial response identification.

Table 6. CPU hours required to create ROM and CFD solutions.

\begin{tabular}{cc|ccc|ccc}
\hline \hline & & \multicolumn{3}{|c|}{ Rigid } & \multicolumn{3}{c}{ Aeroelastic } \\
Model & Case & Static & Dynamic & Total & Static & Dynamic & Total \\
\hline CFD & Linear Forced Rolling Oscillations & 6 & 10,202 & 10,208 & 6 & 10,744 & 10,751 \\
CFD & Linear Forced Yawing Oscillations & 6 & 10,202 & 10,208 & 6 & 10,744 & 10,751 \\
CFD & Linear Forced Pitching Oscillations & 6 & 742 & 748 & 6 & 797 & 803 \\
CFD & Nonlinear Forced Pitching Oscillations & 6 & 742 & 748 & 6 & 797 & 803 \\
\hline ROM & Linear & 6 & 260 & 266 & 6 & 728 & 734 \\
ROM & Nonlinear & 60 & 468 & 528 & 60 & 2,520 & 2,580 \\
\hline \hline
\end{tabular}




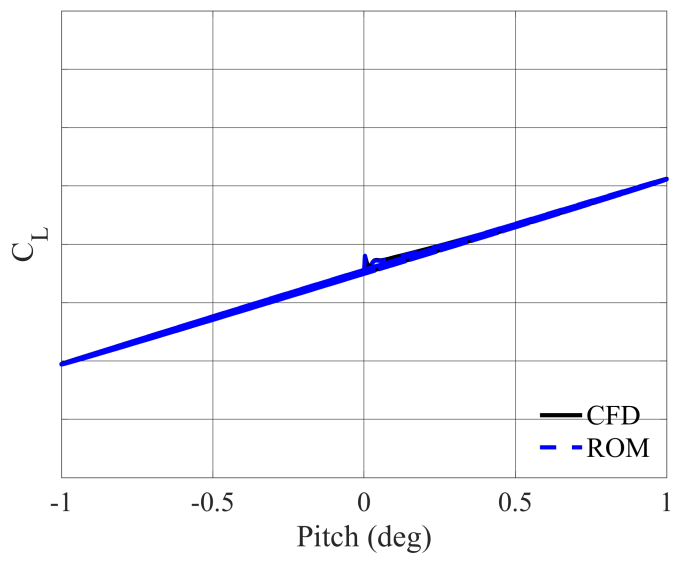

(a) Lift coefficient - Rigid $(k=0.05)$

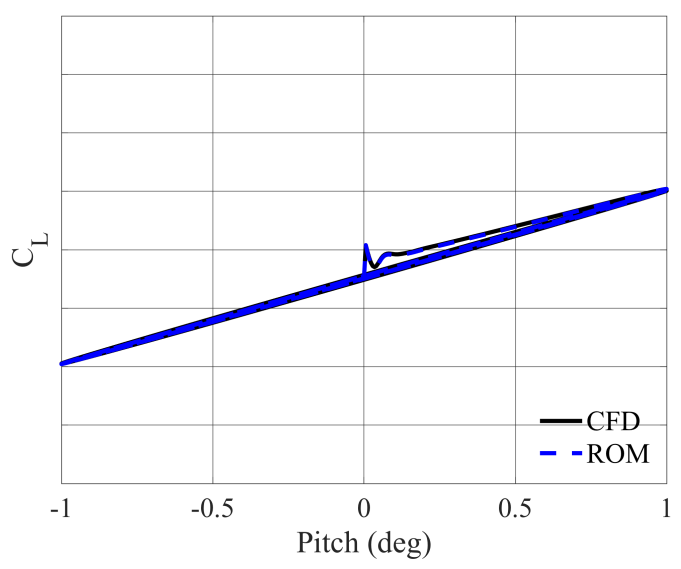

(c) Lift coefficient - Rigid $(k=0.10)$

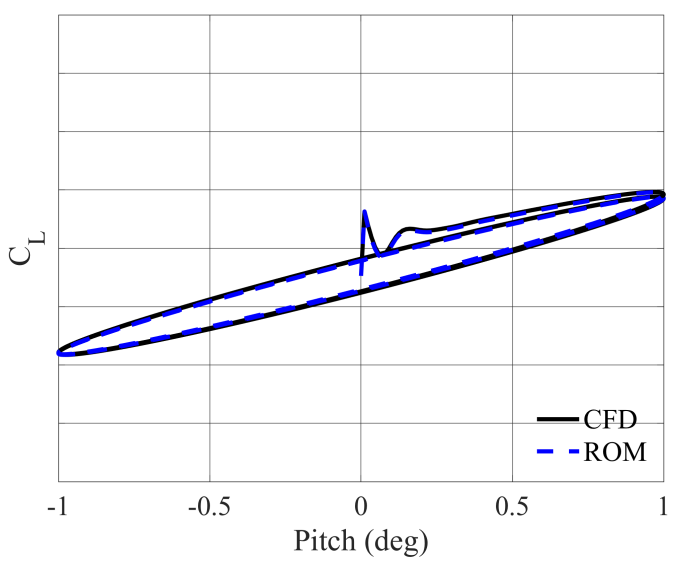

(e) Lift coefficient - Rigid $(k=0.20)$

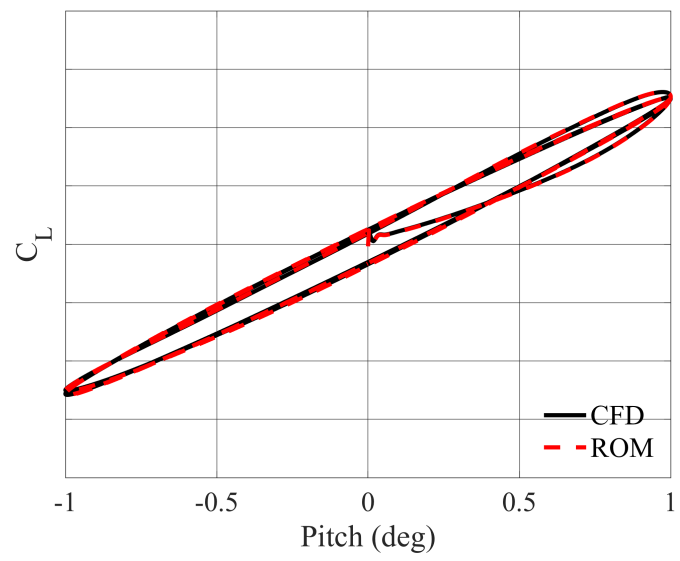

(b) Lift coefficient - Aeroelastic $(k=0.05)$

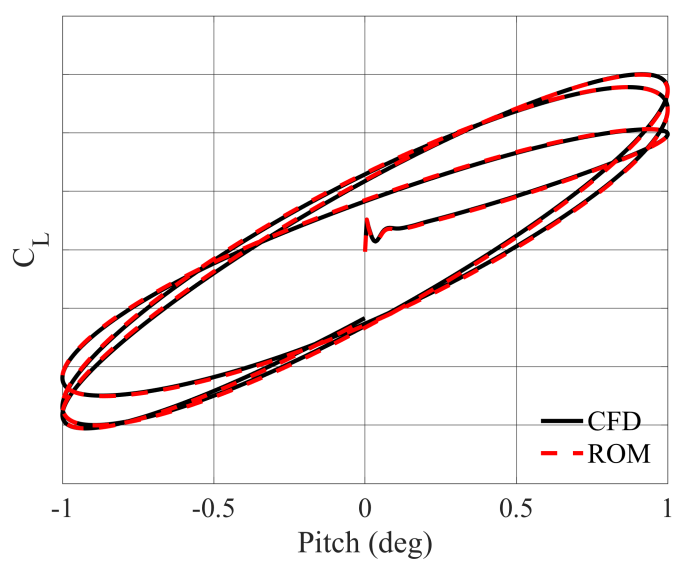

(d) Lift coefficient - Aeroelastic $(k=0.10)$

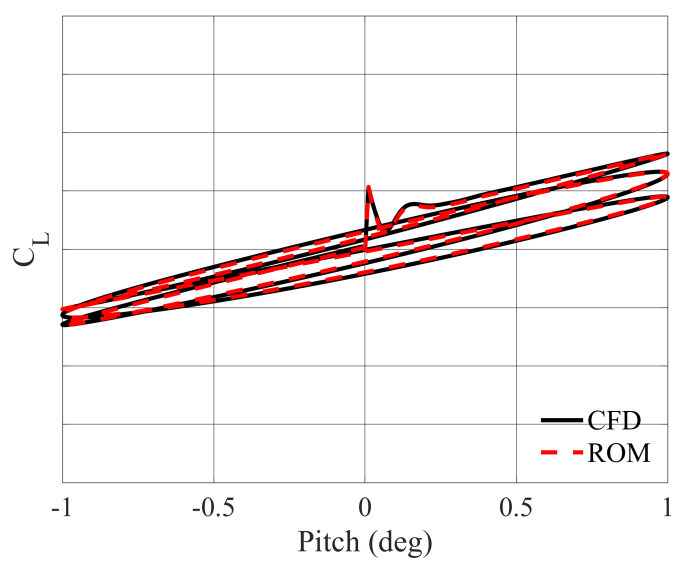

(f) Lift coefficient - Aeroelastic $(k=0.20)$

Figure 20. ROM vs. CFD lift coefficient results for the rigid (left) and flexible (right) X-56A performing a forced pitching oscillation with one degree amplitude for $\mathbf{k}=0.05$ (top), 0.10 (middle), and 0.20 (bottom) at $\alpha=0^{\circ}$, $\beta=\mathbf{0}^{\circ}, \mathbf{M}=\mathbf{0 . 1 3}$. 


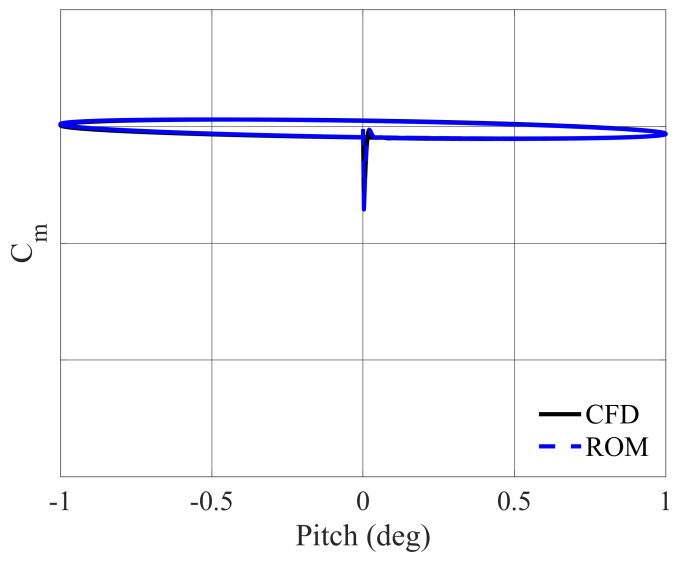

(a) Pitching moment coefficient - Rigid $(k=0.05)$

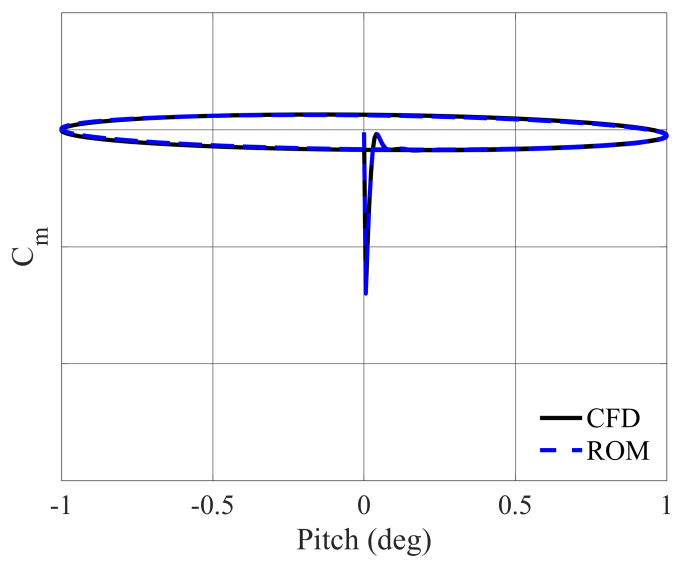

(c) Pitching moment coefficient - Rigid $(k=0.10)$

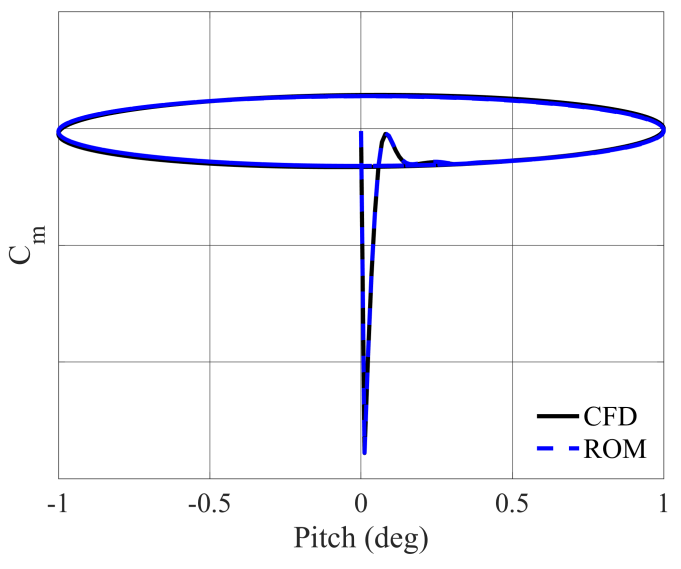

(e) Pitching moment coefficient - Rigid $(k=0.20)$

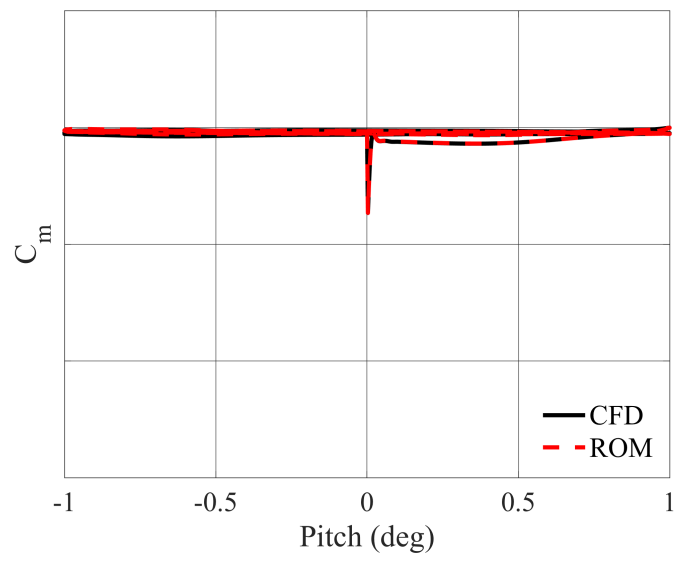

(b) Pitching moment coefficient - Aeroelastic $(k=0.05)$

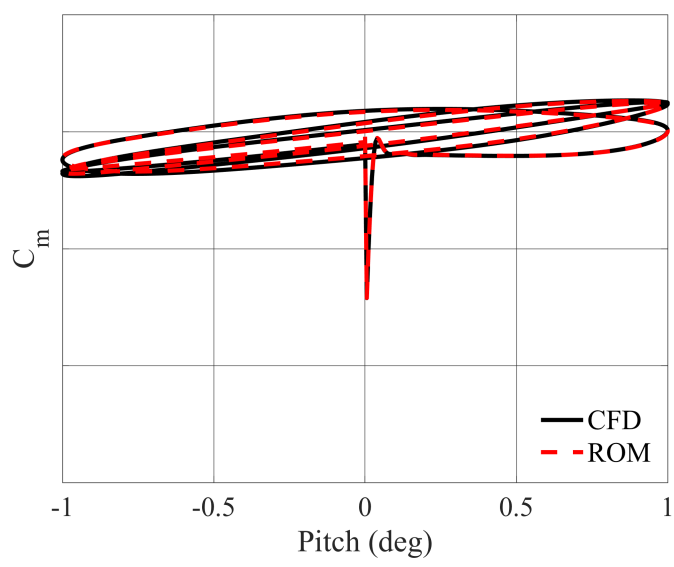

(d) Pitching moment coefficient - Aeroelastic $(k=0.10)$

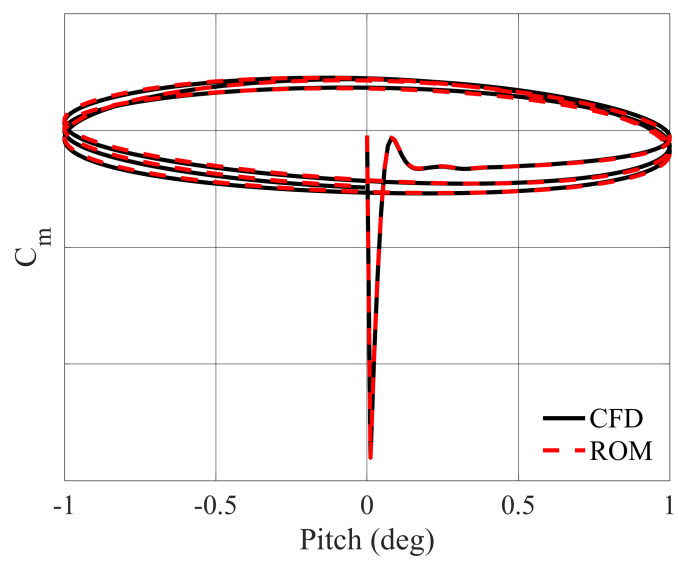

(f) Pitching moment coefficient - Aeroelastic $(k=0.20)$

Figure 21. ROM vs. CFD pitching moment coefficient results for the rigid (left) and flexible (right) X-56A performing a forced pitching oscillation with one degree amplitude for $k=0.05$ (top), 0.10 (middle), and 0.20 (bottom) at $\alpha=\mathbf{0}^{\circ}, \beta=\mathbf{0}^{\circ}, \mathbf{M}=\mathbf{0 . 1 3}$. 


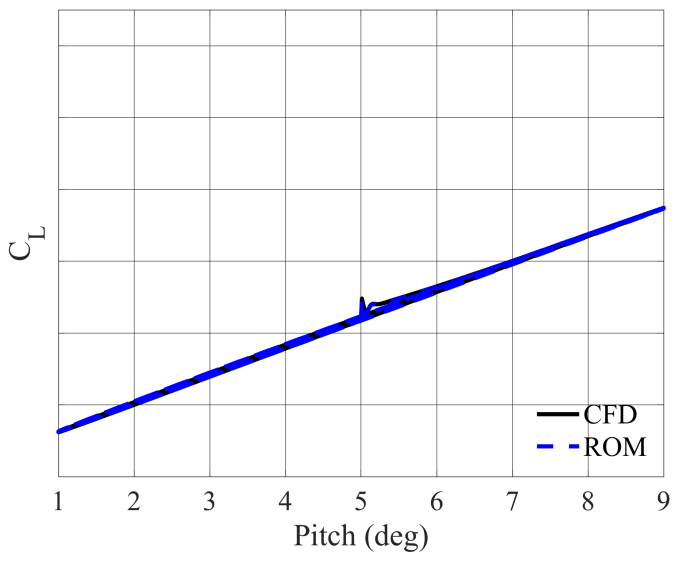

(a) Lift coefficient - Rigid $(k=0.05)$

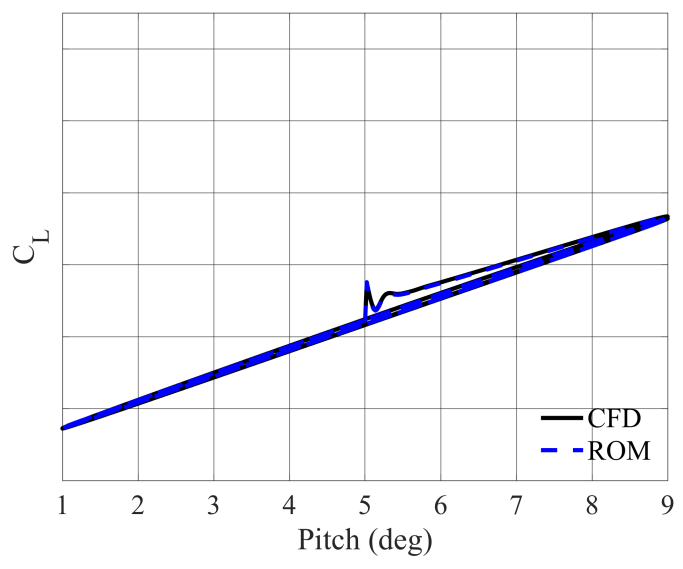

(c) Lift coefficient - Rigid $(k=0.10)$

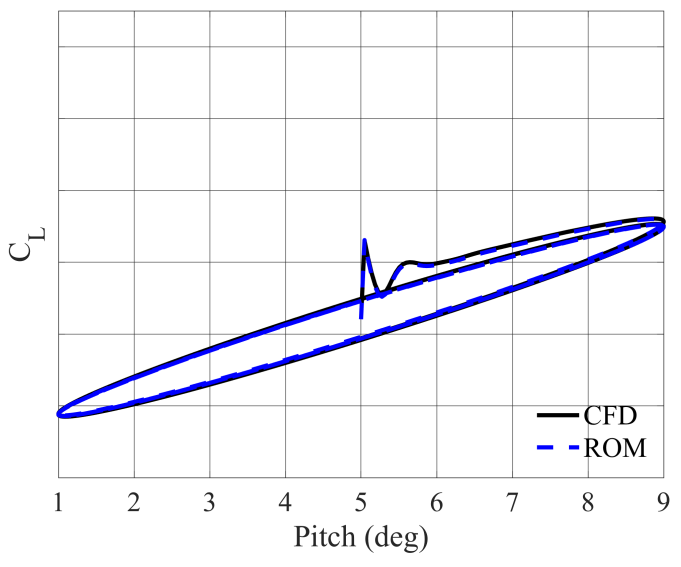

(e) Lift coefficient - Rigid $(k=0.20)$

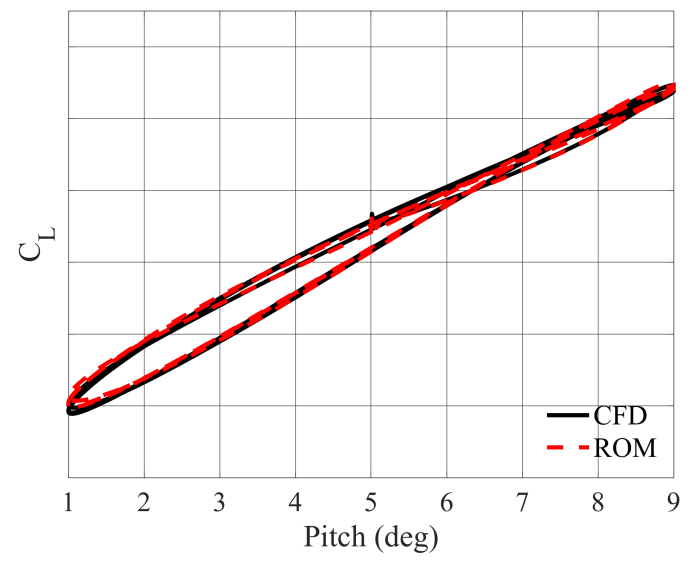

(b) Lift coefficient - Aeroelastic $(k=0.05)$

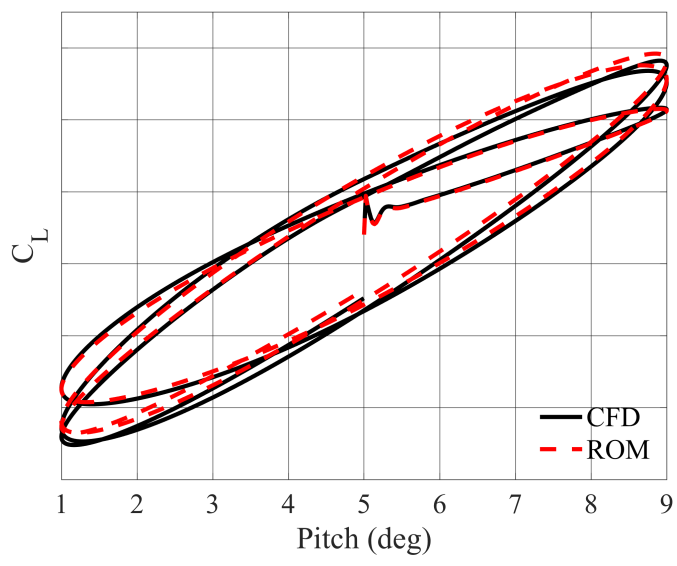

(d) Lift coefficient - Aeroelastic $(k=0.10)$

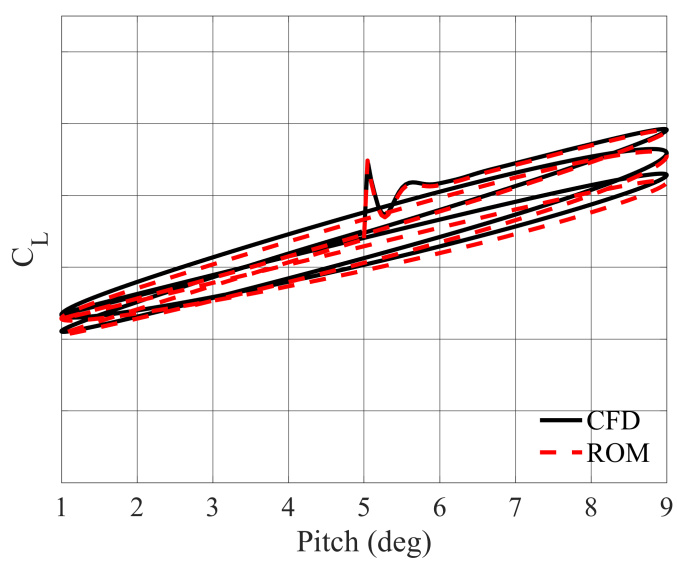

(f) Lift coefficient - Aeroelastic $(k=0.20)$

Figure 22. ROM vs. CFD lift coefficient results for the rigid (left) and flexible (right) X-56A performing a forced pitching oscillation with four degree amplitude for $\mathrm{k}=0.05$ (top), 0.10 (middle), and 0.20 (bottom) at $\alpha=5^{\circ}$, $\beta=\mathbf{0}^{\circ}, \mathbf{M}=\mathbf{0 . 1 3}$. 


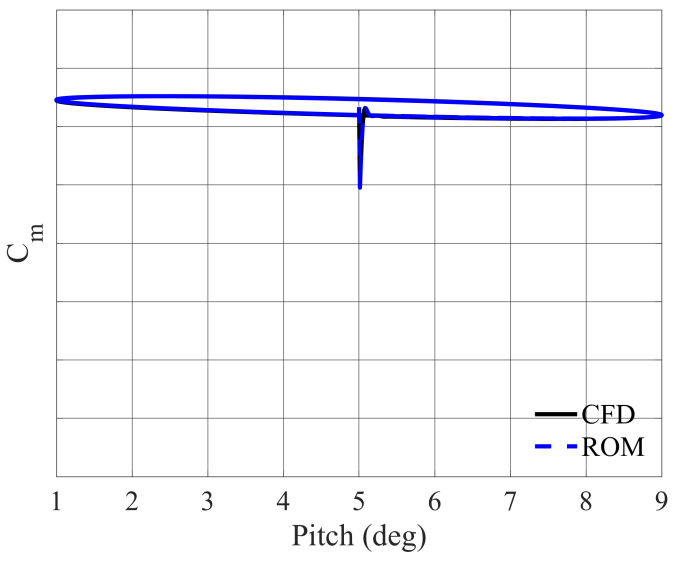

(a) Pitching moment coefficient - Rigid $(k=0.05)$

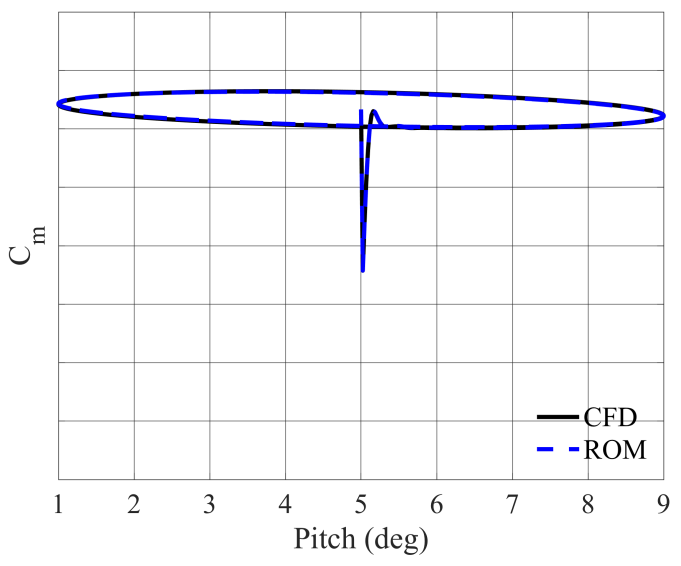

(c) Pitching moment coefficient - Rigid $(k=0.10)$

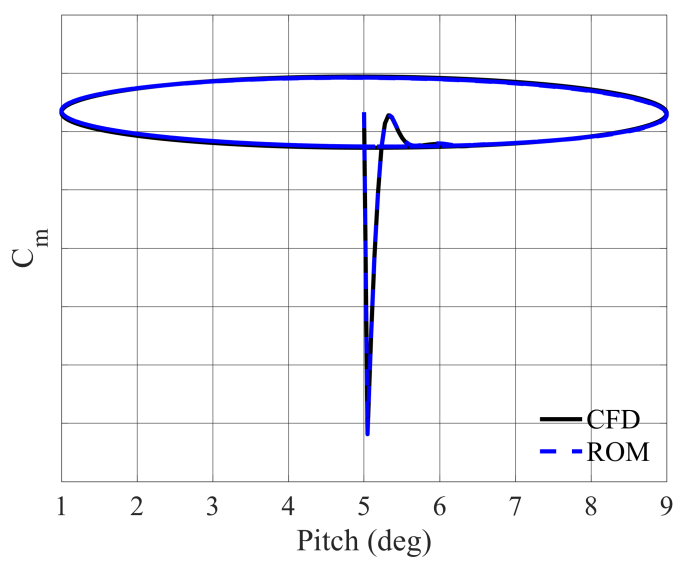

(e) Pitching moment coefficient - Rigid $(k=0.20)$

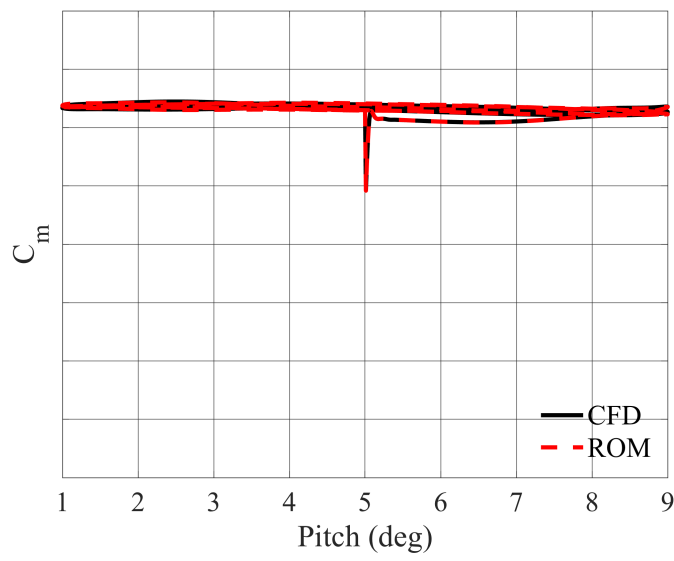

(b) Pitching moment coefficient - Aeroelastic $(k=0.05)$

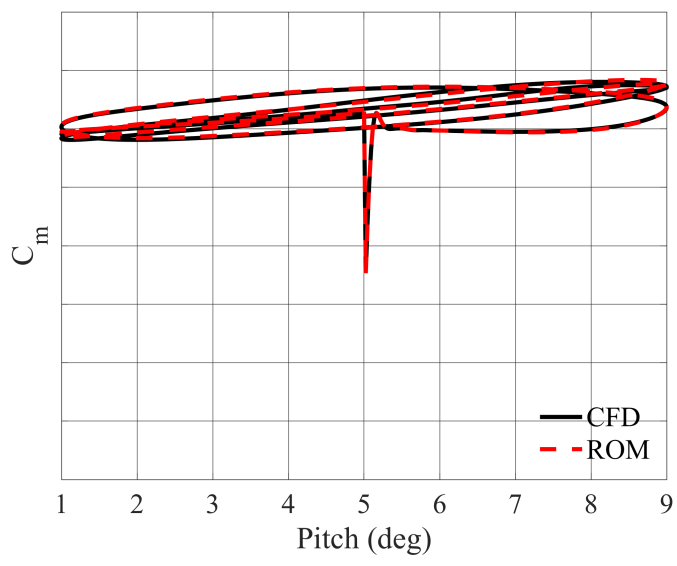

(d) Pitching moment coefficient - Aeroelastic $(k=0.10)$

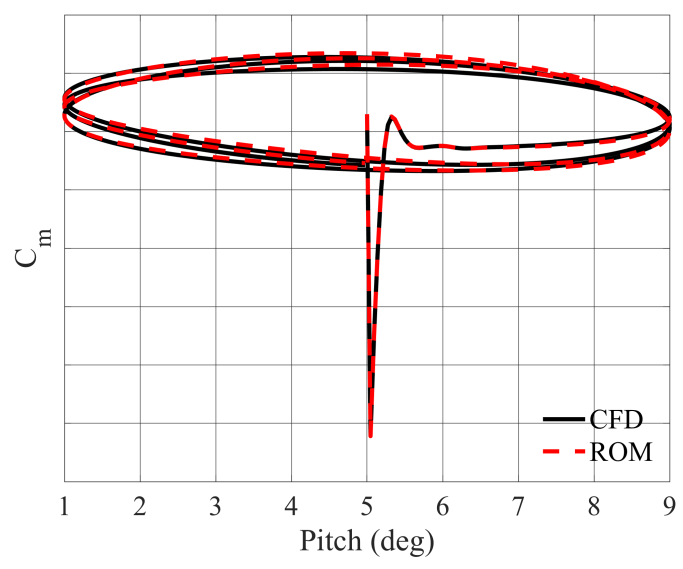

(f) Pitching moment coefficient - Aeroelastic $(k=0.20)$

Figure 23. ROM vs. CFD pitching moment coefficient results for the rigid (left) and flexible (right) X-56A performing a forced pitching oscillation with four degree amplitude for $k=0.05$ (top), 0.10 (middle), and 0.20 (bottom) at $\alpha=5^{\circ}, \beta=0^{\circ}, \mathbf{M}=\mathbf{0 . 1 3}$. 
Overall, the aeroelastic computational costs are only slightly greater than those for the rigid vehicle simulations. This is due to the efficiency of the linear modal structural solver and the use of a single aerostructural coupling iteration per time step. A single time-accurate static initialization solution is required for each forced oscillation. The static initialization solution costs approximately $6 \mathrm{CPU}$ hours for both the rigid and aeroelastic simulations. For the linear and nonlinear ROM, these static initialization costs are multiplied by each of the different initial flow conditions. In the linear case, this is a single solution at $\alpha=0^{\circ}$. For the nonlinear case, this is nine solutions for $\alpha=\left[0^{\circ}\right.$ to $\left.9^{\circ}\right]$.

For each forced oscillation type, the dynamic simulation computational costs are stated for the three frequency cases simulated over three cycles of motion. The lateral forced oscillations require many more iterations relative to the forced pitching oscillation cases as identical reduced frequencies are simulated, but the reference length used to define the motion frequency are based on semispan rather than semichord. Because the X-56A is a high aspect ratio aircraft, this results in a longer simulation period per oscillation cycle. The dynamic simulation costs listed for the rigid and aeroelastic ROMs are based on the costs required for each vehicle-state parameter's indicial response, where 800 iterations are required for rigid response convergence and 4,000 iterations are required for aeroelastic response convergence.

The linear ROM presents comparable cost benefits relative to full-order simulations for both the rigid and aeroelastic cases with nearly 50 times the savings compared to the nine forced oscillation simulations. In contrast, the nonlinear ROM was evaluated only compared to a single nonlinear forced pitching oscillation case. The nonlinear aeroelastic ROM costs present nearly four times the cost of the full-order solution. The utility in using such an approach for efficiently providing unsteady aerodynamic CFD predictions for unconventional vehicle configurations is evident, where the computational benefits scale with the number of evaluations needed. A future publication will demonstrate the aeroelastic indicial response ROM for unsteady aerodynamic predictions of the flexible X-56A performing a right turn maneuver, representative of a flight test conducted by NASA Armstrong. Nonlinear ROM sampling requirements are to be investigated for generalized 6DOF flight trajectories. Additionally, alternative nonlinear ROM methods, such as Volterra theory, should be considered for investigating any nonlinear coupling effects of the vehicle-state parameter inputs, which are not considered in linear indicial response theory.

\section{Conclusions}

The indicial response reduced-order modeling methodology has been evaluated using the NASA FUN3D unstructured flow solver coupled to a linear modal structural solver as a means of efficiently predicting the unsteady aerodynamic response of flexible vehicles maneuvering through arbitrary, prescribed trajectories. Indicial responses with respect to the longitudinal vehicle-state parameters, angle-of-attack and pitch rate, were shown to require over five times the number of time steps relative to rigid simulations in order to converge and resolve the complex, unsteady fluid-structure interactions. Indicial responses with respect to the lateral vehicle-state parameters were shown to be relatively insensitive to flexibility effects. Linear and nonlinear models were generated for the X-56A aircraft undergoing a series of forced rolling, yawing, and pitching oscillations at quasisteady to highly unsteady reduced frequencies. While negligible differences were observed for the rolling and yawing oscillations, the aeroelastic forced pitching oscillations were characterized by cycle-to-cycle variations in aerodynamic damping and minimum/maximum coefficient values at each frequency. The linear and nonlinear ROM results show an ability to capture the initial transients, fluid-structure interactions, and hysteresis behavior for both rigid and aeroelastic simulations. The computational benefits of this modeling approach are shown to scale with the number of maneuver evaluations required for a candidate flight vehicle.

\section{Acknowledgments}

The authors thank Dr. Daniel Reasor of the Air Force Research Laboratory for providing the X56A structural model, as well as Mr. Alexander Chin and Mr. Christopher Acuff of the NASA Armstrong Flight Research Center for providing the X-56A geometry. We would also like to thank the support of Mr. Bob Biedron of the NASA Langley Research Center for his assistance in implementing FUN3D's dynamic aeroelastic analysis capability. 


\section{References}

[1] Morton, S. A., Tillman, B., McDaniel, D. R., Sears, D. R., and Tuckey, T. R., "Kestrel-A Fixed Wing Virtual Aircraft Product of the CREATE Program," DoD High Performance Computing Modernization Program Users Group Conference (HPCMP-UGC), 2009, IEEE, 2009.

[2] Kroll, N., Abu-Zurayk, M., Dimitrov, D., Franz, T., Führer, T., Gerhold, T., Görtz, S., Heinrich, R., Ilic, C., Jepsen, J., et al., "DLR project Digital-X: towards virtual aircraft design and flight testing based on high-fidelity methods," CEAS Aeronautical Journal, Vol. 7, No. 1, 2016.

[3] Lucia, D. J., and Beran, P. S., "Reduced-order model development using proper orthogonal decomposition and volterra theory," AIAA journal, Vol. 42, No. 6, 2004.

[4] Silva, W. A., Haji, M. R., and Prazenica, R. J., "Recent applications of the Volterra theory to aeroelastic phenomena," 23rd Conference and Exposition on Structural Dynamics 2005, IMAC-XXIII, 2005.

[5] Ripepi, M., Verveld, M., Karcher, N., Franz, T., Abu-Zurayk, M., Görtz, S., and Kier, T., "Reduced-order models for aerodynamic applications, loads and MDO,” CEAS Aeronautical Journal, 2018.

[6] Ghoreyshi, M., Jirasek, A., and Cummings, R. M., "Reduced order unsteady aerodynamic modeling for stability and control analysis using computational fluid dynamics,” Progress in Aerospace Sciences, Vol. 71, 2014.

[7] Morton, S. A., McDaniel, D. R., Carlson, H. A., Verberg, R., and Schutt, R., "CFD-Based Reduced-Order Models of F-16XL Static and Dynamic Loads Using Kestrel," 35th AIAA Applied Aerodynamics Conference, 2017.

[8] Suh, P. M., and Mavris, D. N., "Modal Filtering for Control of Flexible Aircraft," 54th AIAA/ASME/ASCE/AHS/ASC Structures, Structural Dynamics, and Materials Conference, 2013.

[9] Suh, P. M., Chin, A. W., and Mavris, D. N., "Robust Modal Filtering and Control fo the X-56A Model with Simulated Fiber Optic Sensor Failures," AIAA Atmospheric Flight Mechanics Conference, 2014.

[10] Suh, P. M., Chin, A. W., and Mavris, D. N., "Virtual Deformation Control of the X-56A Model with Simulated Fiber Optic Sensors,” NASA/TM-2014-216616, NASA Armstrong Flight Research Center, Edwards, CA, 2014.

[11] Mangalam, S. M., and Brenner, M. J., "Fly-by-Feel Sensing and Control: Aeroservoelasticity," AIAA Atmospheric Flight Mechanics Conference, 2014.

[12] Li, W. W., and Pak, C.-G., "Mass Balancing Optimization Study to Reduce Flutter Speeds of the X-56A Aircraft," Journal of Aircraft, 2014. doi:10.2514/1.C033044.

[13] Li, W. W., and Pak, C.-G., "Aeroelastic Optimization Study Based on the X-56A Model," AIAA Atmospheric Flight Mechanics Conference, 2014.

[14] Reasor, D. A., Bhamidipati, K. K., and Chin, A. W., "X-56A Aeroelastic Flight Test Predictions," 54th AIAA Aerospace Sciences Meeting, 2016.

[15] Pak, C.-G., and Truong, S., "Creating a Test Validated Finite-Element Model of the X-56A Aircraft Structure," Journal of Aircraft, 2014. doi:10.2514/1.C033043.

[16] Beranek, J., Nicolai, L., Buonanno, M., Burnett, E., Atkinson, C., Holm-Hansen, B., and Flick, P., "Conceptual Design of a Multi-utility Aeroelastic Demonstrator,” 13th AIAA/ISSMO Multidisciplinary Analysis Optimization Conference, 2010.

[17] Nicolai, L., Hunten, K., Zink, S., and Flick, P., "System Benefits of Active Flutter Suppression for a SensorCraft-Type Vehicle," 13th AIAA/ISSMO Multidisciplinary Analysis Optimization Conference, 2010.

[18] Biedron, R. T., Carlson, J.-R., Derlaga, J. M., Gnoffo, P. A., Hammond, D. P., Jones, W. T., Kleb, B., Lee-Rausch, E. M., Nielsen, E. J., Park, M. A., et al., "FUN3D Manual: 13.3,” 2018.

[19] Biedron, R., and Thomas, J., "Recent enhancements to the FUN3D flow solver for moving-mesh applications," 47th AIAA Aerospace Sciences Meeting including The New Horizons Forum and Aerospace Exposition, 2009.

[20] Frink, N. T., Hiller, B. R., Murphy, P. C., Cunningham, K., and Shah, G. H., "Investigation of Reduced-Order Modeling for Aircraft Stability and Control Prediction," AIAA Scitech 2019 Forum, 2019.

[21] Pak, C.-G., and Truong, S., "Creating a Test Validated Structural Dynamic Finite Element Model of the X-56A Aircraft," 15th AIAA/ISSMO Multidisciplinary Analysis and Optimization Conference, 2014. 
[22] Tobak, M., Chapman, G. T., and Schiff, L. B., "Mathematical modeling of the aerodynamic characteristics in flight dynamics," Proceedings of the Berkeley-Ames Conference on Nonlinear Problems in Control and Fluid Dynamics, Vol. 2, Math Science Pr, 1984.

[23] Tobak, M., and Chapman, G. T., "Nonlinear problems in flight dynamics involving aerodynamic bifurcations," AGARD Symp. on Unsteady Aerodyn. Fundamentals and Appl. to Aircraft Dyn., 1985.

[24] Reisenthel, P., and Reisenthel, P., "Development of a nonlinear indicial model using response functions generated by a neural network," 35th Aerospace Sciences Meeting and Exhibit, 1997.

[25] Reisenthel, P., and Bettencourt, M., "Data-based aerodynamic modeling using nonlinear indicial theory," 37th Aerospace Sciences Meeting and Exhibit, 1997.

[26] Silva, W. A., Discrete-time linear and nonlinear aerodynamic impulse responses for efficient CFD analyses, The College of William and Mary, 1997. PhD Dissertation.

[27] Silva, W. A., "Application of nonlinear systems theory to transonic unsteady aerodynamic responses," Journal of Aircraft, Vol. 30, No. 5, 1993.

[28] Silva, W. A., "Extension of a nonlinear systems theory to general-frequency unsteady transonic aerodynamic responses," 34th Structures, Structural Dynamics and Materials Conference, 1993.

[29] Ghoreyshi, M., Jirásek, A., and Cummings, R. M., "Computational investigation into the use of response functions for aerodynamic-load modeling," AIAA journal, Vol. 50, No. 6, 2012.

[30] Ghoreyshi, M., and Cummings, R., "Aerodynamics modeling of a maneuvering aircraft using indicial functions," 50th AIAA Aerospace Sciences Meeting including the New Horizons Forum and Aerospace Exposition, 2012.

[31] Ghoreyshi, M., and Cummings, R. M., "Unsteady aerodynamics modeling for aircraft maneuvers: A new approach using time-dependent surrogate modeling," Aerospace Science and Technology, Vol. 39, 2014.

[32] Ghoreyshi, M., Cummings, R. M., and Lofthouse, A., "Sampling Strategies for Reduced-Order Modeling of Nonlinear and Unsteady Aerodynamics," 52nd Aerospace Sciences Meeting, 2014.

[33] Ghoreyshi, M., Frink, N. T., van Rooij, M., Lofthouse, A. J., Cummings, R. M., and Nayani, S., "Collaborative Evaluation of CFD-to-ROM Dynamic Modeling," 54th AIAA Aerospace Sciences Meeting, 2016.

[34] Sears, W. R., "Some aspects of non-stationary airfoil theory and its practical application," Journal of the Aeronautical Sciences, Vol. 8, No. 3, 1941. 